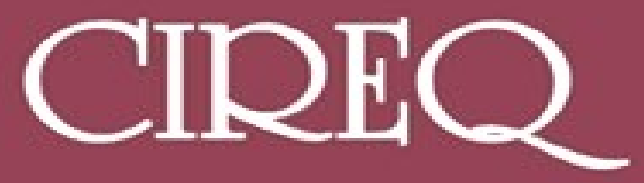

Cahier 06-2006

SHARING THE COST OF A PUBLIC GOOD: AN INCENTIVE-CONSTRAINED AXIOMATIC APPROACH

François MANIQUET and Yves SPRUMONT 


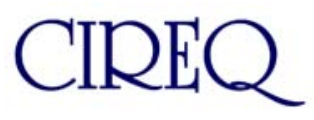

Le Centre interuniversitaire de recherche en économie quantitative (CIREQ) regroupe des chercheurs dans les domaines de l'économétrie, la théorie de la décision, la macroéconomie et marchés financiers, la microéconomie appliquée et économie expérimentale et l'économie de l'environnement et des ressources naturelles. Ils proviennent principalement des universités de Montréal, McGill et Concordia. Le CIREQ offre un milieu dynamique de recherche en économie quantitative grâce au grand nombre d'activités qu'il organise (séminaires, ateliers, colloques) et de collaborateurs qu'il reçoit chaque année.

The Center for Interuniversity Research in Quantitative Economics (CIREQ) regroups researchers in the fields of econometrics, decision theory, macroeconomics and financial markets, applied microeconomics and experimental economics, and environmental and natural resources economics. They come mainly from the Université de Montréal, McGill University and Concordia University. CIREQ offers a dynamic environment of research in quantitative economics thanks to the large number of activities that it organizes (seminars, workshops, conferences) and to the visitors it receives every year.

\section{Cahier 06-2006}

\section{SHARING THE COST OF A PUBLIC GOOD: AN INCENTIVE-CONSTRAINED AXIOMATIC APPROACH}

François MANIQUET and Yves SPRUMONT

CIREQ, Université de Montréal C.P. 6128 , succursale Centre-ville Montréal (Québec) H3C 3J7 Canada téléphone : (514) 343-6557 télécopieur : (514) 343-5831 cireq@umontreal.ca http://www.cireq.umontreal.ca
Université th de Montréal
McGill

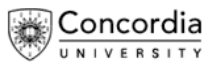


Ce cahier a également été publié par le Département de sciences économiques de l'Université de Montréal sous le numéro 2006-09.

This working paper was also published by the Department of Economics of the University of Montreal under number 2006-09.

Dépôt légal - Bibliothèque nationale du Canada, 2006, ISSN 0821-4441

Dépôt légal - Bibliothèque et Archives nationales du Québec, 2006

ISBN-13 : 978-2-89382-528-1; ISBN-10 : 2-89382-528-1 


\title{
Sharing the cost of a public good: An incentive-constrained axiomatic approach
}

\author{
François Maniquet*and Yves Sprumont ${ }^{\dagger}$
}

June 15, 2006

\begin{abstract}
We study the problem of provision and cost-sharing of a public good in large economies where exclusion, complete or partial, is possible. We search for incentive-constrained efficient allocation rules that display fairness properties. Population monotonicity says that an increase in population should not be detrimental to anyone. Demand monotonicity states that an increase in the the demand for the public good (in the sense of a first-order stochatic dominance shift in the distribution of preferences) should not be detrimental to any agent whose preferences remain unchanged.

Under suitable domain restrictions, there exists a unique incentiveconstrained efficient and demand-monotonic allocation rule: the so-called serial rule. In the binary public good case, the serial rule is also the only incentive-constrained efficient and population-monotonic rule.
\end{abstract}

Keywords: excludable public good, incentive compatibility, fairness, serial rule

JEL classification numbers: D63, D71

${ }^{*}$ CORE, Université Catholique de Louvain, voie du Roman Pays 34, B-1348 Louvain-laNeuve, Belgium (maniquet@core.ucl.ac.be)

${ }^{\dagger}$ Département de Sciences Économiques and CIREQ, Université de Montréal, C.P. 6128, succursale centre-ville, Montréal QC, H3C 3J7, Canada (yves.sprumont@umontreal.ca) 


\section{Introduction}

We study the problem of provision and cost-sharing of a public good when exclusion, complete or partial, is possible: the good can be consumed at different levels by different agents. Examples include television channels, uncongested sport facilities or public transportation systems, and so on. Binary public goods, where consumption is either zero or one, are a particular and much studied case.

Ideally, a solution to this problem should be efficient, fair, and incentivecompatible. Several approaches are found in the literature.

A first stream of research, following Lindahl (1928), ignores the incentive issue and aims at designing a fair and efficient production and cost-sharing plan for each possible configuration of preferences. Different allocation rules are possible, such as Kaneko's (1977) ratio equilibrium and its generalization by Mas-Colell (1980), or Moulin's (1987) egalitarian-equivalent rule and Sprumont's (1998) variant. These solutions are first-best efficient (implying that no agent is ever excluded from the consumption of the good) and satisfy different fairness properties. But they are manipulable: truthful revelation is not a dominant strategy in the associated direct revelation game where agents report their preferences and the allocation rule is applied to the reported profile. Each individual agent has an interest to under-represent her taste for the good: this is the well-known free-rider problem.

The incentive issue can be handled indirectly by constructing game forms implementing the desired solution rule, either in Nash equilibrium for rules à la Lindahl, Kaneko, Mas-Colell (see Hurwicz (1979), Walker (1981) among many others) or in subgame-perfect equilibrium for Moulin's egalitarian-equivalent rule or Sprumont's variant (see Crawford (1979), Demange (1984), Maniquet (2003)). The complete information requirement underlying the Nash and subgame-perfect equilibrium concepts makes these mechanisms of very limited use in large economies.

A second line of research addresses the incentive compatibility problem directly by concentrating on the requirement of strategy-proofness: truthful revelation of one's preference should be a dominant strategy in the direct revelation game associated with the allocation rule. Unless one accepts grossly unfair (essentially dictatorial) rules, the price to pay is in terms of efficiency.

The literature has identified two families of interesting strategy-proof rules achieving limited forms of efficiency. For quasi-linear preferences, the celebrated Clarke-Groves mechanisms (see among others Clarke (1971), Groves and Ledyard (1977), Green and Laffont (1979)) are strategy-proof, yield the efficient level of 
production of the public good, and do not exclude any agent from its consumption. But they may charge more than the total production cost - thus falling short of full efficiency - and may leave low-valuation agents with a net welfare loss, violating the so-called participation constraint.

Starting with Drèze (1980), the more recent literature has underlined the benefits of allowing for exclusion. The possibility of excluding agents who report a low valuation of the public good alleviates the free-rider problem and allows to balance the budget without violating the participation constraints. In particular, the serial mechanism (Moulin (1994), Dearden (1998), Olszewski (2004)) is strategyproof (even in the coalitional sense and without the quasi-linearity assumption), budget-balanced, satisfies the participation constraints, and Pareto-dominates any mechanism possessing these properties. Of course, it involves exclusion and fails to produce the efficient level of the public good. Norman (2004) argues for simpler mechanisms setting a level of the public good, giving full access to each agent willing to pay a fixed fee, and producing the good whenever the contributions cover the cost.

The welfare losses of the two types of mechanisms - budget surplus in the Clarke-Groves mechanisms, underconsumption in the mechanisms involving exclusionare difficult to compare: see Moulin (1994), Deb, Gosh and Seo (2002), and, for the case of a binary public good, Moulin and Shenker (2001) and Mutuswami (2004). Neihter the Clarke-Groves mechanisms nor the mechanisms involving exclusion have been much studied from a distributive viewpoint.

A third approach defines for each economy a social ordering of the allocations and maximizes it subject to the relevant feasibility and incentive compatibility constraints. Hellwig (2005) uses a utilitarian criterion; Maniquet and Sprumont $(2004,2005)$ axiomatize ordinal maxmin criteria. This second-best approach has a long tradition in public economics, particularly in taxation theory (initiated by Mirrlees (1971), see Diamond (1998) for a recent updating). Provided that the social ordering satisfies the Pareto principle (that is, unanimously preferred allocations are better), maximizing it yields allocations that cannot be Paretodominated by any other incentive-compatible allocation. The more recent literature emphasizes fairness concerns (see Fleurbaey and Maniquet (2006) for an overview). These concerns are incorporated by imposing restrictions on the social ordering rather than on the chosen allocation.

This approach has the benefit of coherence: social choices arise from the maximization, under different informational or institutional restrictions, of the same social ordering. One drawback is technical: due to the nature of the incentive 
compatibility constraints, the relevant maximization problem is often hard to solve. A second criticism is that the choice correspondence resulting from the maximization of the chosen ordering under varying constraints may exhibit poor or counter-intuitive properties. Fairness can be defined in terms of social goals or in terms of actual choices; it turns out that the two views are not in complete harmony.

Motivated by this difficulty, the current paper suggests a fourth approach. In each economy, we restrict our attention, from the outset, to the technologically feasible and incentive-compatible allocations — with or without exclusion. We search for allocation rules that i) select allocations that are incentive-constrained efficient (that is, Pareto-undominated among the feasible and incentive-compatible allocations) and ii) display attractive fairness properties.

As we consider economies with a continuum of agents, incentive compatibility is properly expressed by the no-envy requirement. Our definition of feasibility assumes that the distribution of preferences is known to the planner. This is the informational framework of optimal taxation theory à la Mirrlees. ${ }^{1}$

The fairness properties we consider are participation, population monotonicity, and demand monotonicity. Participation requires that no agent be worse off than when consuming no public good and paying nothing. Population monotonicity requires that if population increases, no agent initially present in the economy should be worse off. This property is ethically appealing when none of the initial agents bears any particular responsibility for the arrival of the newcomers: equal treatment then requires that all should be affected in the same way and, since the produced good is public in nature, making all of them worse off would go against our ethical intuition. Population monotonicity also provides the correct incentives to welcome newcomers: after all, they lower average cost. Demand monotonicity is in the same spirit. It states that an increase in the demand for the public good - in the sense of a first-order stochastic shift in the distribution of preferences should not harm any agent whose preferences are unchanged. Again, since none of these agents is responsible for the good news that the others are willing to pay more for the public good, they should all be affected in the same direction. Because of the public nature of the good, they should all gain from the increase.

Population monotonicity and demand monotonicity are examples of solidarity axioms. Such axioms have been used extensively in the theory of fair alloca-

\footnotetext{
${ }^{1}$ In finite economies, the proper formulation of incentive-compatibility would be strategyproofness and the planner should not be assumed to know the distribution of preferences. This makes the entire analysis much more complex.
} 
tion (see Thomson (2004) for a survey). The novelty of this paper is to combine them with the requirement of incentive-constrained efficiency. We show that there is a unique incentive-constrained efficient allocation rule satisfying demand monotonicity. This rule, called the serial rule, is also population-monotonic and, in the particular case of binary public goods, is the unique population-monotonic incentive-constrained efficient allocation rule. It works as follows: each unit of the public good is successively made available at a price computed so that the revenue collected from the agents willing to pay that price is precisely equal to the incremental cost of producing that unit. Production stops when no price allows to cover the incremental cost. Agents select their preferred consumption level, possibly zero, and pay the corresponding bill. If we think of agents with a low willingness to pay as poor, the rule guarantees that the poor do not contribute to cover the cost of the units they cannot consume. At the same time, the rich agents do not subsidize the poor: they would be worse off in their absence.

For expositional reasons, we first derive our result under simple but restrictive assumptions. Preferences are linear and the revenue function, which determines how much revenue the planner can collect by charging any given price, is strictly concave whenever it is increasing. The last section generalizes the result to quasi-linear economies satisfying the Spence-Mirrlees single-crossing property and a suitable extension of the concave revenue assumption. All proofs are gathered in the appendix.

\section{The model}

We consider an environment with one private good, hereafter called money, and one public good, to which we refer as the good. The good can be produced at any integer level $y \in \mathbb{N}=\{0,1, \ldots\}$. Producing $y$ units costs $x=C(y)$ units of money. The cost function $C: \mathbb{N} \rightarrow \mathbb{R}_{+}$is fixed throughout; we write $c(y)=C(y)-C(y-1)$ for all $y \in \mathbb{N} \backslash\{0\}$ and define $c(0)=0$. We assume that costs are strongly convex in the following sense: $C(0)=0<c(y)<c(y+1)$ for all $y \in \mathbb{N} \backslash\{0\}$, and the mapping $c$ is unbounded.

An agent's preference is an ordering over bundles consisting of a consumption level of the good $y \in \mathbb{N}$ and a monetary contribution or cost share $x \in \mathbb{R}$. We assume that this preference is monotonic and linear. It may therefore be represented by a single number $v \in \mathbb{R}_{+}$expressing the agent's valuation of any one unit of the good: $v y-x$ measures her welfare from the bundle $(x, y)$.

The economies we consider are large and anonymous. We model them as 
cumulative distribution functions over the preference space. Formally, a (linear) economy is a pair $(I, E)$ where $I=[\underline{v}, \bar{v}] \subset \mathbb{R}_{+}$is a non-degenerate interval of valuations and $E: \mathbb{R}_{+} \rightarrow[0,1]$ is continuous, strictly increasing on $I$, and such that $E(\underline{v})=0$ and $E(\bar{v})=1$. For any $v \in I, E(v)$ represents the proportion of agents whose valuation of the good does not exceed $v$. Continuity of $E$ guarantees that the proportion of agents with any given valuation is zero. Strict monotonicity guarantees that there is a positive proportion of agents with a valuation in any non-degenerate subinterval of $I$.

For brevity, we sometimes do not mention the valuation interval $I$ and call $E$ itself an economy. We call $J \subseteq I$ an $\left(E\right.$-)positive set if $\int_{J} d E>0$, an (E-)full set if $\int_{J} d E=1$. A statement is true for $(E$-)almost all $v \in I$ if it is true for all $v$ in a full set $J \subseteq I$.

\section{Incentive compatibility and feasibility}

Throughout Sections 3 to $5,(I, E)$ is a fixed economy. We consider a planner whose knowledge of the environment is limited to $C$, the available technology, and $(I, E)$, the distribution of preferences. Observe that this latter piece of information is anonymous: the planner ignores the preferences of individual agents.

Definition 1. An allocation (for $(I, E)$ ) is a pair $(\mathbf{x}, \mathbf{y})$, where $\mathbf{x}: I \rightarrow \mathbb{R}$ is an integrable mapping specifying a (possibly negative) cost share $\mathbf{x}(v)$ for each (agent reporting) valuation $v$, and $\mathbf{y}: I \rightarrow \mathbb{N}$ is a mapping determining the corresponding levels of consumption of the good. This allocation is incentive-compatible if

$$
v \mathbf{y}(v)-\mathbf{x}(v) \geq v \mathbf{y}\left(v^{\prime}\right)-\mathbf{x}\left(v^{\prime}\right) \text { for all } v, v^{\prime} \in I .
$$

Let $A(I, E)$ denote the set of incentive-compatible allocations for $(I, E)$.

Incentive compatibility is the fundamental constraint implied by the planner's informational handicap. If $v \mathbf{y}(v)-\mathbf{x}(v)<v \mathbf{y}\left(v^{\prime}\right)-\mathbf{x}\left(v^{\prime}\right)$, then an agent with valuation $v$ finds it profitable to report a valuation $v^{\prime}$. Since the planner is unable to detect such misreports, he must avoid them.

Our first task is to describe the incentive-compatible allocations. This is conveniently done by means of pricing schemes.

Definition 2. A pricing scheme (for $(I, E)$ ) is a pair $(a, p)$, where $a \in \mathbb{R}$ and $p: \mathbb{N} \backslash\{0\} \rightarrow \mathbb{R}_{+}$is such that $p(y) \geq \bar{v}$ for all $y$ sufficiently large. 
The number $a$ is a fixed, perhaps negative, monetary contribution and $p(y)$ is the price charged for consuming the $y$ th unit of the good. An agent consuming $y$ units thus pays a cost share $a+\sum_{q=1}^{y} p(q) .^{2}$

We say that the pricing scheme $(a, p)$ generates (or represents) the allocation $(\mathbf{x}, \mathbf{y})$ if every agent with valuation $v$ maximizes her welfare level $v y-(a+$ $\left.\sum_{q=1}^{y} p(q)\right)$ by choosing $y=\mathbf{y}(v)$, and the resulting cost share $a+\sum_{q=1}^{\mathbf{y}(v)} p(q)$ coincides with $\mathbf{x}(v)$. The restriction that unit prices eventually reach the highest valuation in the economy guarantees that each agent's maximization problem has a solution.

As the following example illustrates, different pricing schemes may represent the same incentive-compatible allocation.

Example 1. Let $v^{\mu}=\int v d E$ denote the mean valuation in the economy $(I, E)$. Let $y^{\mu}$ be the smallest maximizer of $v^{\mu} y-C(y)$ over all $y \in \mathbb{N}$ and define $\mathbf{y}^{\mu}(v)=y^{\mu}$ and $\mathbf{x}^{\mu}(v)=C\left(y^{\mu}\right)$ for all $v \in I$. This surplus-maximizing allocation $\left(\mathbf{x}^{\mu}, \mathbf{y}^{\mu}\right)$ is trivially incentive-compatible.

Here are three different pricing schemes representing $\left(\mathbf{x}^{\mu}, \mathbf{y}^{\mu}\right)$. Fix $\overline{\bar{v}}>\bar{v}$. In the first scheme, $a^{1}=C\left(y^{\mu}\right), p^{1}(y)=0$ for all $y \leq y^{\mu}$, and $p^{1}(y)=\overline{\bar{v}}$ for all $y>y^{\mu}$. In the second scheme, $a^{2}=C\left(y^{\mu}\right)-\underline{v} y^{\mu}, p^{2}(y)=\underline{v}$ for all $y \leq y^{\mu}$, and $p^{2}(y)=\bar{v}$ for all $y>y^{\mu}$. In the third scheme, unit prices fail to be nondecreasing: $a^{3}=C\left(y^{\mu}\right)$, $p^{3}(y)=0$ for all $y \leq y^{\mu}, p^{3}\left(y^{\mu}+1\right)=\overline{\bar{v}}$, and $p^{3}(y)=\bar{v}$ for all $y>y^{\mu}+1$.

In order to obtain a standard representation, we impose further restrictions. ${ }^{3}$

Definition 3. A pricing scheme $(a, p)$ is nondecreasing if $p$ is a nondecreasing mapping. It is standard if it is nondecreasing and $p(y) \in I$ for all $y \in \mathbb{N} \backslash\{0\}$.

\section{Theorem 1.}

(a) If $(a, p)$ is a nondecreasing pricing scheme, then any allocation $(\mathbf{x}, \mathbf{y})$ for $(I, E)$ satisfying

$$
\mathbf{y}(v) \in\{y \in \mathbb{N} \mid p(y) \leq v \leq p(y+1)\}
$$

and

$$
\mathbf{x}(v)=a+\sum_{y=1}^{\mathbf{y}(v)} p(y)
$$

for all $v \in I$ is incentive-compatible.

\footnotetext{
${ }^{2}$ Here and throughout the paper, we adopt the convention that a sum defined over the empty set is zero.

${ }^{3}$ Other standardizations are possible; the one proposed here is particularly handy when studying Pareto dominance: see Lemma 1 below.
} 
(b) If $(\mathbf{x}, \mathbf{y})$ is an incentive-compatible allocation for $(I, E)$, then there exists a unique standard pricing scheme (a,p) satisfying (3.2) and (3.3).

Condition (3.2) says that every agent with valuation $v$ maximizes her welfare level $v y-\left(a+\sum_{q=1}^{y} p(q)\right)$ by choosing $y=\mathbf{y}(v)$. Condition (3.3) says that the resulting cost share coincides with $\mathbf{x}(v)$, thereby ensuring that $(a, p)$ represents $(\mathbf{x}, \mathbf{y})$.

Statement (a) thus asserts that every allocation generated by an arbitrary nondecreasing pricing scheme is incentive-compatible. That statement is obvious: since all agents maximize their welfare under the same constraints, no one can possibly prefer someone else's bundle.

If $(a, p)$ generates two incentive-compatible allocations $(\mathbf{x}, \mathbf{y}),\left(\mathbf{x}^{\prime}, \mathbf{y}^{\prime}\right)$, these allocations coincide on almost all valuations and are Pareto-equivalent: $v \mathbf{y}(v)-$ $\mathbf{x}(v)=v \mathbf{y}^{\prime}(v)-\mathbf{x}^{\prime}(v)$ for all $v \in I$. To check these claims, observe that whenever $p(y)<v<p(y+1)$ for some $y \in \mathbb{N}$, that level $y$ is necessarily unique and (3.2) forces $\mathbf{y}(v)=y$. Thus, the only agents whose bundle is not fully determined are those with a valuation $v$ equal to some unit price $p(y)$. By assumption, their proportion in the population is zero. For these valuations $v$, several consumption levels $\mathbf{y}(v)$ are compatible with (3.2) but the associated welfare level $v \mathbf{y}(v)-\mathbf{x}(v)$, where $\mathbf{x}(v)$ is given by (3.3), is unique.

Statement (b) says that every incentive-compatible allocation is represented by a unique standard pricing scheme. The proof of this statement is not entirely obvious; it relies crucially on the linearity of preferences.

A useful property of any incentive-compatible allocation $(\mathbf{x}, \mathbf{y})$ follows at once from statement (b): both $\mathbf{x}$ and $\mathbf{y}$ are nondecreasing in $v$. Because $I$ is a closed interval, it follows that both $\mathbf{x}$ and $\mathbf{y}$ must have a finite range and $\inf _{v \in I} \mathbf{y}(v)=$ $\mathbf{y}(\underline{v})$ and $\sup _{v \in I} \mathbf{y}(v)=\mathbf{y}(\bar{v})$. We write $\mathbf{y}(\underline{v})=\underline{y}, \mathbf{y}(\bar{v})=\bar{y}$, and call $\bar{y}$ the production level at $(\mathbf{x}, \mathbf{y})$. Two incentive-compatible allocations generated by the same standard pricing scheme $(a, p)$ need not have the same production level since (3.2) does not determine $\mathbf{y}(\bar{v})$ uniquely.

We now turn to the second constraint faced by the planner: the selected allocation must be technologically feasible.

Definition 4. An allocation $(\mathbf{x}, \mathbf{y})$ for $(I, E)$ is feasible if it satisfies the constraint

$$
\int_{I} \mathbf{x} d E \geq C\left(\sup _{v \in I} \mathbf{y}(v)\right) .
$$

Let $A^{C}(I, E)$ denote the set of feasible incentive-compatible allocations for $(I, E)$. 
The left-hand side of inequality (3.4) is the revenue expected from the allocation $(\mathbf{x}, \mathbf{y})$; the right-hand side is its cost. Both can be computed by the planner as he knows $(I, E)$.

If $(\mathbf{x}, \mathbf{y})$ is incentive-compatible, its cost is equal to $C(\bar{y}) \cdot{ }^{4}$ The revenue actually collected coincides with the expected revenue and Theorem 1 provides us with a convenient expression for it. If $(\mathbf{x}, \mathbf{y})$ is represented by the nondecreasing pricing scheme $(a, p)$, then $\int_{I} \mathbf{x} d E=a+\int_{I} \sum_{y=1}^{\mathbf{y}(v)} p(y) d E=a+\sum_{y=1}^{\bar{y}} p(y) \int_{p(y)}^{\bar{v}} d E=$ $a+\sum_{y=1}^{\bar{y}} p(y)(1-E(p(y)))=a+\sum_{y=1}^{\infty} p(y)(1-E(p(y)))$.

Define the revenue function associated with $E, r(., E): \mathbb{R}_{+} \rightarrow \mathbb{R}$, by

$$
r(v, E)=v(1-E(v))
$$

for all $v \in \mathbb{R}_{+}$. Note that $r(v, E)=v$ for all $v \leq \underline{v}$ and $r(v, E)=0$ for all $v \geq \bar{v}$. We write $r$ for $r(., E)$ whenever the reference to $E$ is clear. Using this notation,

$$
\int_{I} \mathbf{x} d E=a+\sum_{y=1}^{\bar{y}} r(p(y))=a+\sum_{y=1}^{\infty} r(p(y)) .
$$

This formula shows that all allocations generated by a given nondecreasing pricing scheme $(a, p)$ yield exactly the same total revenue even though they may involve different production levels, hence different production costs.

Having defined incentive compatibility and feasibility, we conclude this section with a brief illustration of the classical second-best approach -the third approach described in the introduction. Given $E$, define the utilitarian ordering $\mathbf{R}(E)$ over all (not necessarily incentive-compatible or feasible) allocations by

$$
(\mathbf{x}, \mathbf{y}) \mathbf{R}(E)\left(\mathbf{x}^{\prime}, \mathbf{y}^{\prime}\right) \Leftrightarrow \int_{I}(v \mathbf{y}(v)-\mathbf{x}(v)) d E \geq \int_{I}\left(v \mathbf{y}^{\prime}(v)-\mathbf{x}^{\prime}(v)\right) d E .
$$

Maximizing this ordering subject to incentive compatibility and feasibility yields the surplus-maximizing allocation $\left(\mathbf{x}^{\mu}, \mathbf{y}^{\mu}\right)$ of Definition 1 . Because $\mathbf{R}(E)$ satisfies the Pareto principle, $\left(\mathbf{x}^{\mu}, \mathbf{y}^{\mu}\right)$ cannot be Pareto-dominated by any feasible

\footnotetext{
${ }^{4}$ This statement requires a qualification. Incentive compatibility does not rule out that $\bar{v} \mathbf{y}(\bar{v})-\mathbf{x}(\bar{v})=\bar{v} \mathbf{y}(v)-\mathbf{x}(v)$ for some $v$ such that $\mathbf{y}(v)<\mathbf{y}(\bar{v})$. In that case, the agents with valuation $\bar{v}$ do not have a strict incentive to report truthfully. If they report valuation $v$, the resulting maximal consumption generates a cost $C(\mathbf{y}(v))<C(\mathbf{y}(\bar{v}))=C(\bar{y})$. Thus $C(\bar{y})$ is the cost of $(\mathbf{x}, \mathbf{y})$ at the truthful dominant-strategy equilibrium of the revelation game but there may be other dominant-strategy equilibria generating a lower cost. However, we will see below that the cost of an efficient incentive-compatible allocation is unambiguous.
} 
incentive-compatible allocation ${ }^{5}$. This allocation violates all three equity properties discussed in the introduction: participation, population monotonicity, and demand monotonicity. In particular, the low-valuation agents are typically worseoff than when consuming no public good and paying nothing.

\section{Serial allocations}

The so-called serial allocations are an important example of incentive-compatible allocations. They are generated by the following pricing scheme. Let $Y^{s}$ be the set of all production levels $y \in \mathbb{N}$ such that

$$
r(v, E) \geq c(y)
$$

for some $v \in \mathbb{R}_{+}$. Our assumptions guarantee that there is a maximal level $y^{s} \in \mathbb{N}$ such that $Y^{s}=\left\{0, \ldots, y^{s}\right\}$. For every $y \in Y^{s} \backslash\{0\}$, let $v^{s}(y)$ be the smallest valuation $v \in \mathbb{R}_{+}$such that (4.1) is true: $v^{s}(y)$ is well defined because $E$ is continuous. Extend the mapping $v^{s}$ by defining $v^{s}(y)=\overline{\bar{v}}$ for all $y>y^{s}$, where $\overline{\bar{v}}>\bar{v}$. By construction,

$$
r\left(v^{s}(y), E\right)=c(y)
$$

for all $y \in Y^{s} \backslash\{0\}$, that is, charging a price $v^{s}(y)$ for unit $y$ guarantees that the revenue collected from the agents whose valuations exceed that price covers exactly the cost of producing that unit. Note also that $v^{s}(y)<v^{s}(y+1)$ for every $y \in Y^{s}$.

The pair $\left(0, v^{s}\right)$ is a nondecreasing pricing scheme. The serial allocations for $(I, E)$ are the allocations that are Pareto-equivalent to those generated by $\left(0, v^{s}\right)$ and have the same production level, $y^{s}$. For a formal description, let $y_{s}$ be the largest $y \in \mathbb{N}$ such that $c(y) \leq \underline{v}$ and let $a^{s}=C\left(y_{s}\right)-\underline{v} y_{s}$. For each $y \in \mathbb{N} \backslash\{0\}$, define $w^{s}(y)=\max \left\{\underline{v}, v^{s}(y)\right\}$. Notice that $\left(a^{s}, w^{s}\right)$ coincides with $\left(0, v^{s}\right)$ if and only if $\underline{v} \leq c(1)$.

Definition 5. A serial allocation for $(I, E)$ is any allocation $(\mathbf{x}, \mathbf{y})$ generated by the pricing scheme $\left(a^{s}, w^{s}\right)$, that is, satisfying

$$
\mathbf{y}^{s}(v) \in\left\{y \in \mathbb{N} \mid w^{s}(y) \leq v \leq w^{s}(y+1)\right\}
$$

\footnotetext{
${ }^{5}$ In fact, this allocation is not Pareto-dominated by any feasible allocation, whether incentivecompatible or not. This first-best efficiency property is irrelevant when incentive-compatibility is taken seriously: allocations that are not incentive-compatible are not an option for the planner.
} 
and

$$
\mathbf{x}^{s}(v)=a^{s}+\sum_{y=1}^{\mathbf{y}^{s}(v)} w^{s}(y)
$$

for all $v \in I$.

From Theorem 1 and the ensuing discussion, serial allocations are essentially unique: they coincide on almost all valuations and are Pareto-equivalent. The revenue collected covers exactly the associated cost of production ${ }^{6}: \int_{I} \mathbf{x}^{s} d E=$ $a^{s}+\sum_{y=1}^{y^{s}} r\left(w^{s}(y), E\right)=C\left(y_{s}\right)+\sum_{y=y_{s}+1}^{y^{s}} c(y)=C\left(y^{s}\right)$.

A word of clarification is in order. The pricing scheme $\left(a^{s}, w^{s}\right)$ is not quite standard since $w^{s}(y)>\bar{v}$ for all $y>y^{s}$. This is important: together with (4.3), it forces $\bar{y}^{s}:=\mathbf{y}^{s}(\bar{v})$ to coincide with $y^{s}$. We call this number the serial production level in the economy $(I, E)$. Under the alternative standard convention that prices are bounded above by $\bar{v}$, condition (4.3) would allow $\mathbf{y}^{s}(\bar{v})>y^{s}$. Other allocations would then qualify as serial, all Pareto-equivalent to the ones defined here but differing in the bundle assigned to the agents with valuation $\bar{v}$. They would generate the same revenue but a higher production cost. Choosing $w^{s}(y)>\bar{v}$ for all $y>y^{s}$ guarantees feasibility.

Of course, according to Theorem 1 (b), every serial allocation $\left(\mathbf{x}^{s}, \mathbf{y}^{s}\right)$ for $(I, E)$ is representable by a unique standard scheme, which we denote $\left(a^{s}, p^{s}\right)$ and call the standard serial pricing scheme for $(I, E): p^{s}(y)=w^{s}(y)$ for $y=1, \ldots, y^{s}$ and $p^{s}(y)=\bar{v}$ for $y>y^{s}$. As explained above, this scheme generates all the serial allocations and other allocations that are Pareto-equivalent, revenue-equivalent, but not cost-equivalent to them.

\section{Efficiency}

From now on, we restrict our attention to $A(I, E)$, the set of incentive-compatible allocations. This section studies efficiency in the second-best sense: we are interested in the feasible incentive-compatible allocations that are not Paretodominated by any feasible incentive-compatible allocation.

Definition 6. An allocation $\left(\mathbf{x}^{\prime}, \mathbf{y}^{\prime}\right) \in A(I, E)$ Pareto-dominates an allocation $(\mathbf{x}, \mathbf{y}) \in A(I, E)$ if $v \mathbf{y}^{\prime}(v)-\mathbf{x}^{\prime}(v) \geq v \mathbf{y}(v)-\mathbf{x}(v)$ for all $v \in I$ and this inequality is strict for all $v$ in an $E$-positive set.

\footnotetext{
${ }^{6}$ By construction, $v^{s}\left(y^{s}\right)<\bar{v}$ if $y^{s}>0$. This implies that all dominant-strategy equilibria of the revelation game yield the production level $y^{s}$ and the resulting cost is $C\left(y^{s}\right)$, avoiding the difficulty explained in Footnote 4.
} 
An allocation $(\mathbf{x}, \mathbf{y}) \in A^{C}(I, E)$ is efficient if there is no $\left(\mathbf{x}^{\prime}, \mathbf{y}^{\prime}\right) \in A^{C}(I, E)$ which Pareto-dominates it.

In order to describe the efficient allocations in $A^{C}(I, E)$, we begin with a characterization of Pareto dominance. If $(a, p),\left(a^{\prime}, p^{\prime}\right)$ are nondecreasing pricing schemes representing two incentive-compatible allocations $(\mathbf{x}, \mathbf{y}),\left(\mathbf{x}^{\prime}, \mathbf{y}^{\prime}\right)$, we write $\left(a^{\prime}, p^{\prime}\right) \preceq(a, p)$ if and only if $a^{\prime}+\sum_{q=1}^{y} p^{\prime}(q) \leq a+\sum_{q=1}^{y} p(q)$ for all $y \in \mathbb{N}$. We write $\left(a^{\prime}, p^{\prime}\right) \prec(a, p)$ if and only if $\left(a^{\prime}, p^{\prime}\right) \preceq(a, p)$ and $\left(a^{\prime}, p^{\prime}\right) \neq(a, p)$ : the total contribution required for consuming any (possibly zero) level of the good is never higher, and sometimes lower, under the pricing scheme $\left(a^{\prime}, p^{\prime}\right)$ than under $(a, p)$.

Lemma 1. Let $(\mathbf{x}, \mathbf{y}),\left(\mathbf{x}^{\prime}, \mathbf{y}^{\prime}\right) \in A(I, E)$ be represented by their standard pricing schemes $(a, p),\left(a^{\prime}, p^{\prime}\right)$. Then $\left(\mathbf{x}^{\prime}, \mathbf{y}^{\prime}\right)$ Pareto-dominates $(\mathbf{x}, \mathbf{y})$ if and only if $\left(a^{\prime}, p^{\prime}\right) \prec(a, p)$.

It is important that both pricing schemes in this lemma are standard. Example 1 shows that the same incentive-compatible allocation may be represented by two pricing schemes $(a, p),\left(a^{\prime}, p^{\prime}\right)$ such that $\left(a^{\prime}, p^{\prime}\right) \prec(a, p)$.

Next we must take the feasibility constraint (3.4) into account: total revenue must cover production cost. Here we face a difficulty. Consider two incentivecompatible allocations $(\mathbf{x}, \mathbf{y}),\left(\mathbf{x}^{\prime}, \mathbf{y}^{\prime}\right)$ with identical production levels $\bar{y}=\bar{y}^{\prime}$, represented by their standard pricing schemes $(a, p),\left(a^{\prime}, p^{\prime}\right)$. It may happen that $\left(a^{\prime}, p^{\prime}\right) \prec(a, p)$, yet $a^{\prime}+\sum_{y=1}^{\bar{y}} r\left(p^{\prime}(y), E\right)>a+\sum_{y=1}^{\bar{y}} r(p(y), E):$ more revenue is collected by asking a lower contribution for any consumption level of the good.

\section{Example 2.}

Consider an economy $(I, E)$, where $I=\left[0, \frac{3}{2}\right]$ and

$$
\begin{aligned}
E(v) & =v \text { if } 0 \leq v \leq \frac{1}{2}, \\
& =\frac{1}{4}+\frac{1}{2} v \text { if } \frac{1}{2} \leq v \leq \frac{3}{2} .
\end{aligned}
$$

On $I$, the associated revenue function is $r(v)=v(1-v)$ if $0 \leq v \leq \frac{1}{2}$ and $r(v)=\frac{v}{2}\left(\frac{3}{2}-v\right)$ if $\frac{1}{2} \leq v \leq \frac{3}{2}$. As Figure 5.1a shows, this function is the maximum of two concave (quadratic) functions. It is strictly increasing on $\left[0, \frac{3}{4}\right]$ and strictly decreasing on $\left[\frac{3}{4}, \frac{3}{2}\right]$. It is differentiable everywhere but at $\frac{1}{2}$ : its left derivative at that point is zero while its right derivative is positive. This implies in particular that the function is not concave.

Suppose $c(1)=\frac{1}{4}<c(2)<\frac{9}{32}<c(3)$. Then the serial production level is $y^{s}=2$ and the standard serial pricing scheme gives $a^{s}=0$ and $p^{s}(1)=\frac{1}{2}<p^{s}(2)<\frac{3}{4}$, 
$p^{s}(y)=\frac{3}{2}$ for all $y \geq 3$. Consider an alternative standard pricing scheme $(a, p)$ such that $a=0, p(1)=p^{s}(1)-\varepsilon, p(2)=p^{s}(2)+\varepsilon$, and $p(y)=p^{s}(y)$ for all $y \geq 3$, where $\varepsilon>0$. By construction $(a, p) \prec\left(a^{s}, p^{s}\right)$. See Figure 5.1b. Because the left derivative of $r$ is zero at $p^{s}(1)$ and its derivative is positive at $p^{s}(2)$, the revenue generated by $(a, p)$ is higher than that generated by $\left(a^{s}, p^{s}\right)$ for any $\varepsilon$ small enough. Thus, more revenue is collected by asking a lower contribution for any consumption level of the good. This example also shows that the serial allocations are not always efficient.

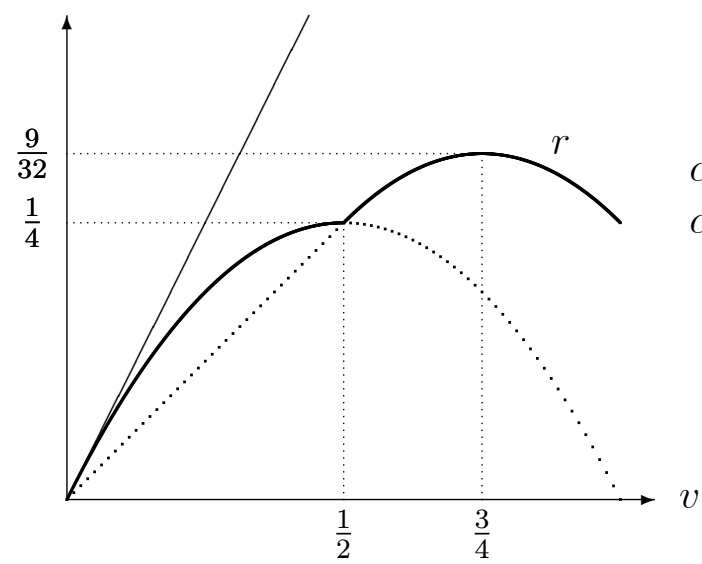

(a)

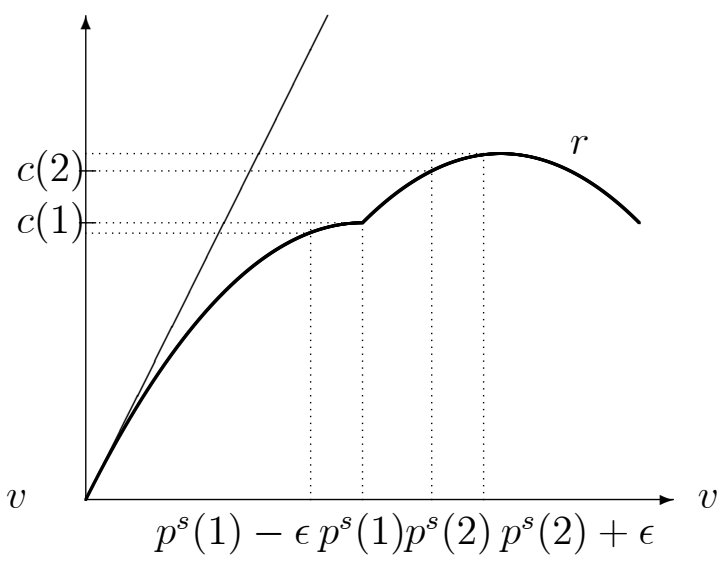

(b)

Figure 5.1: Charging less may increase revenue

The possibility of collecting more revenue by charging less for every consumption level of the good in the interval of prices where the revenue function is increasing strikes us as implausible. It also makes a tractable description of the efficient allocations in $A^{C}(I, E)$ virtually impossible as feasible Pareto improvements are extremely difficult to identify. We introduce an assumption on $(I, E)$ that rules it out.

Assumption L1. The revenue function $r(., E)$ is strictly concave on $\left[\underline{v}, v^{*}\right]$, where $v^{*}$ is the smallest valuation maximizing $r(., E)$. 
The assumption is violated by the economy in Example 2. Large classes of economies satisfy it, however.

\section{Example 3.}

1) Any economy $E$ generating a symmetric single-peaked density on, say, $I=[0,1]$, satisfies Assumption L1. Suppose, for simplicity, that $E$ is twice differentiable. Strict concavity of the associated revenue function at $v$ means $r^{\prime \prime}(v)<0$, that is, $2 E^{\prime}(v)+v E^{\prime \prime}(v)>0$. This condition is obviously satisfied for all $v \in\left(0, \frac{1}{2}\right)$. Now check that $r$ is decreasing on $\left[\frac{1}{2}, 1\right]:$ compute $r^{\prime}(v)=1-E(v)-v E^{\prime}(v)$ and observe that if $v \in\left[\frac{1}{2}, 1\right)$, symmetry entails $(1-E(v))-v E^{\prime}(v)=E(1-v)-$ $v E^{\prime}(1-v) \leq E(1-v)-(1-v) E^{\prime}(1-v) \leq 0$.

2) As a second illustration, consider an economy generating a triangular density on $I=[0,1]$ :

$$
\begin{aligned}
E(v) & =\frac{v^{2}}{\alpha} \text { if } 0 \leq v \leq \alpha, \\
& =1+\alpha-\frac{(1-v)^{2}}{1-\alpha} \text { if } \alpha \leq v \leq 1,
\end{aligned}
$$

where $\alpha \in[0,1]$. The associated revenue function is $r(v)=v-\frac{v^{3}}{\alpha}$ if $0 \leq v \leq \alpha$ and $r(v)=\frac{v(1-v)^{2}}{1-\alpha}-\alpha v$ if $\alpha \leq v \leq 1$.

We claim that $E$ satisfies Assumption L1 if $\alpha \in\left[\frac{1}{3}, 1\right]$. It is easy to check that $r$ is strictly concave on $[0, \alpha]$ for any $\alpha \in[0,1]$. Next, $r$ is strictly decreasing on $[\alpha, 1]$ whenever $\alpha \in\left[\frac{1}{3}, 1\right]: r^{\prime}(v)=\frac{(1-v)(1-3 v)}{1-\alpha}-\alpha<0$ for $\alpha<v<1$. The claim follows.

We are now ready to prove the following key result.

Lemma 2. Suppose $(I, E)$ satisfies Assumption L1 and let $(a, p),\left(a^{\prime}, p^{\prime}\right)$ be nondecreasing pricing schemes for $(I, E)$. Fix $\bar{y} \in \mathbb{N} \backslash\{0\}$ such that $p(\bar{y}) \leq v^{*}$.

Then $\left(a^{\prime}, p^{\prime}\right) \preceq(a, p)$ implies $a^{\prime}+\sum_{y=1}^{\bar{y}} r\left(p^{\prime}(y), E\right) \leq a+\sum_{y=1}^{\bar{y}} r(p(y), E)$. If $(a, p),\left(a^{\prime}, p^{\prime}\right)$ are standard, then $\left(a^{\prime}, p^{\prime}\right) \prec(a, p)$ implies $a^{\prime}+\sum_{y=1}^{\bar{y}} r\left(p^{\prime}(y), E\right)<$ $a+\sum_{y=1}^{\bar{y}} r(p(y), E)$.

Under Assumption L1, Lemmas 1 and 2 deliver the following corollary. At an incentive-compatible allocation where revenue equals cost and the standard price of the last unit produced does not exceed the smallest revenue-maximizing price, no Pareto improvement is possible without changing the production level. 
Using this fact, the theorem below provides a set of four easy-to-check conditions that are necessary and sufficient for any feasible incentive-compatible allocation $(\mathbf{x}, \mathbf{y})$ to be efficient under Assumption L1. The first condition is budget balance: revenue equals production cost. The second condition is that the production level is not below the serial level. The remaining conditions apply if production is positive. The third says that the price of the last unit produced does not exceed the smallest revenue-maximizing price. ${ }^{7}$ The fourth essentially amounts to checking that one particular incentive-compatible allocation Pareto-dominating $(\mathbf{x}, \mathbf{y})$ is not feasible. If $(a, p)$ is the standard pricing scheme representing $(\mathbf{x}, \mathbf{y})$, find the unique $(\preceq-)$ maximal standard pricing scheme $\left(a^{\prime}, p^{\prime}\right)$ such that $\left(a^{\prime}, p^{\prime}\right) \preceq(a, p)$ and

$$
a^{\prime}+\sum_{y=1}^{\bar{y}-1} p^{\prime}(y) \leq a+\sum_{y=1}^{\bar{y}} p(y)-\bar{v} .
$$

This scheme ${ }^{8}$, denoted by $\left(a^{1}, p^{1}\right)$, is illustrated in Figure 5.2. The condition says that $\left(a^{1}, p^{1}\right)$ does not generate enough revenue to cover the cost of producing $\bar{y}-1$ units of the good.

Theorem 2. Suppose $(I, E)$ satisfies Assumption L1 and let $y^{s}$ be the serial production level in this economy. An allocation $(\mathbf{x}, \mathbf{y}) \in A^{C}(I, E)$ represented by its standard pricing scheme $(a, p)$ is efficient if and only if

1) $a+\sum_{y=1}^{\bar{y}} r(p(y), E)=C(\bar{y})$

2) $\bar{y} \geq y^{s}$

3) $p(\bar{y}) \leq v^{*}$

4) $a^{1}+\sum_{y=1}^{\bar{y}-1} r\left(p^{1}(y), E\right)<C(\bar{y}-1)$, where the last two conditions apply whenever they are defined, that is, if $\bar{y}>0$.

As an illustration of Theorem 2, the following example describes a one-parameter infinite family of efficient allocations.

\footnotetext{
${ }^{7}$ This condition implies that $p(\bar{y})<\bar{v}$. It follows that $\bar{y}$ is the production level at all dominantstrategy equilibria of the revelation game. See Footnote 4.

${ }^{8}$ The pricing sheme $\left(a^{1}, p^{1}\right)$ may be computed as follows. Let $w^{1}$ be the smallest valuation $v \in I$ such that $v(\bar{y}-1)-\left(a+\sum_{y=1}^{\bar{y}} p(y)-\bar{v}\right) \geq v \mathbf{y}(v)-\mathbf{x}(v)$. If $w^{1}<\bar{v}$, let $a^{1}=a$ and $p^{1}(y)=$ $\min \left\{p(y), w^{1}\right\}$ if $y \leq \bar{y}-1, p^{1}(y)=\bar{v}$ if $y \geq \bar{y}$. If $w^{1}=\bar{v}$, let $a^{1}=\left(a+\sum_{y=1}^{\bar{y}} p(y)-\bar{v}\right)-\underline{v}(\bar{y}-1)$ and $p^{1}(y)=\underline{v}$ if $y \leq \bar{y}-1, p^{1}(y)=\bar{v}$ if $y \geq \bar{y}$.
} 


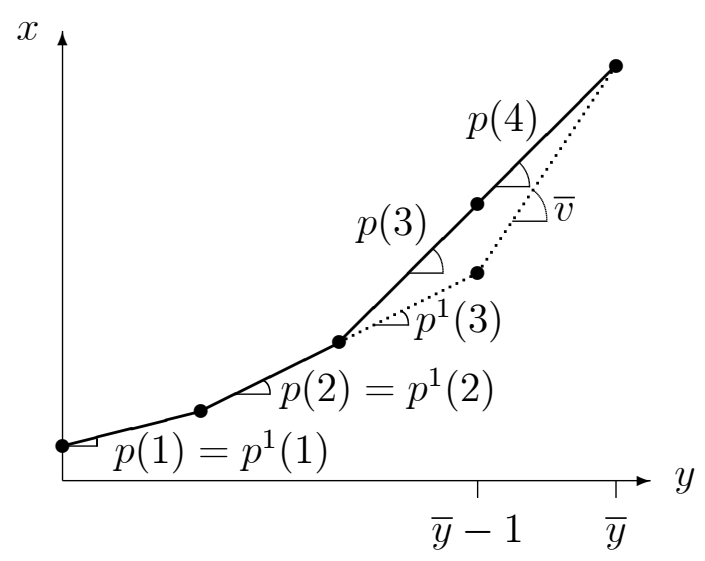

Figure 5.2: Condition 4 of theorem 2: constructing $\left(a^{1}, p^{1}\right)$ from $(a, p)$

Example 4. For any $v^{\alpha} \in I$, let $y^{\alpha}$ be the smallest maximizer of $v^{\alpha} y-C(y)$ over all $y \in \mathbb{N}$ and define the following standard pricing scheme: $a^{\alpha}=C\left(y^{\alpha}\right)-\underline{v} y^{\alpha}$ and $p^{\alpha}(y)=\underline{v}$ if $y \leq y^{\alpha}, p^{\alpha}(y)=p^{s}(y)$ if $y>y^{\alpha}$. Let $\left(\mathbf{x}^{\alpha}, \mathbf{y}^{\alpha}\right)$ be generated by $\left(a^{\alpha}, p^{\alpha}\right)$ and such that $\bar{y}^{\alpha}=\max \left\{y^{\alpha}, y^{s}\right\}$.

If $v^{\alpha}=\underline{v},\left(\mathbf{x}^{\alpha}, \mathbf{y}^{\alpha}\right)$ is a serial allocation. If $v^{\alpha}=v^{\mu}$, it is a cost-minimal surplus-maximizing allocation (see Example 1). It is routine to check that all four conditions in Theorem 2 are met.

\section{An axiomatic defense of serial allocation rules}

This section explores incentive-compatible allocations across economies with the aim of designing efficient and fair allocation rules. Let $\mathcal{L}$ be the set of (linear) economies. An (allocation) rule on a domain $\mathcal{D} \subseteq \mathcal{L}$ is a mapping $\varphi$ assigning to each $(I, E) \in \mathcal{D}$ an allocation $\varphi(I, E) \in A^{C}(I, E)$. We say that a rule $\varphi$ is serial if, for every $(I, E) \in \mathcal{D}, \varphi(I, E)$ is a serial allocation for $(I, E)$.

Our main contribution is an axiomatic defense of the serial allocation rules. First, we require efficiency in the sense of Section 5.

Efficiency. For all $(I, E) \in \mathcal{D}, \varphi(I, E)$ is efficient.

Turning to distributive concerns, we seek to formulate a proper definition of egalitarianism. One possible interpretation would consist of equalizing consumption bundles: all agents would consume the same level of the good and pay the 
same cost share. This interpretation is unappealing for the following reason. According to condition 2 in Theorem 2, efficiency requires that the public good be provided at a high enough (above serial) level. Therefore, unless the interval of valuations $I$ is very small, agents with a low valuation of the public good are typically worse off at an egalitarian efficient allocation than if they do not participate in the production and cost-sharing mechanism.

A second possible interpretation of egalitarianism is to equalize opportunity sets: all agents choose from the same menu of consumption bundles. This requirement, equivalent to No Envy (Kolm (1966)), is automatically met since we restrict our attention to incentive-compatible allocations. See Theorem 1.

A third possibility is to require that agents be equally affected by changes that they have not initiated. Following this line of interpretation, we will examine two axioms: demand monotonicity and population monotonicity. Demand monotonicity requires that when some agents in an economy become richer, the welfare of all the others varies in the same direction. More precisely, all the agents whose valuation of the public good is unchanged should benefit from an increase in the willingness to pay of the others.

If $\left(I^{1}, E^{1}\right),\left(I^{2}, E^{2}\right) \in \mathcal{D}$, let us write $\left(I^{1}, E^{1}\right) \unlhd\left(I^{2}, E^{2}\right)$ if and only if $E^{2}(v) \leq E^{1}(v)$ for all $v \in \mathbb{R}_{+}$. For any given price, the proportion of agents with a valuation higher than that price is larger in $\left(I^{2}, E^{2}\right)$ than in $\left(I^{1}, E^{1}\right)$, that is, $\left(I^{2}, E^{2}\right)$ first-order stochastically dominates $\left(I^{1}, E^{1}\right)$. We think of $\left(I^{2}, E^{2}\right)$ as a "richer" economy.

Demand monotonicity. If $\left(I^{1}, E^{1}\right),\left(I^{2}, E^{2}\right) \in \mathcal{D},\left(I^{1}, E^{1}\right) \unlhd\left(I^{2}, E^{2}\right), \varphi\left(I^{1}, E^{1}\right)=$ $\left(\mathbf{x}^{1}, \mathbf{y}^{1}\right)$ and $\varphi\left(I^{2}, E^{2}\right)=\left(\mathbf{x}^{2}, \mathbf{y}^{2}\right)$, then $v \mathbf{y}^{2}(v)-\mathbf{x}^{2}(v) \geq v \mathbf{y}^{1}(v)-\mathbf{x}^{1}(v)$ for all $v \in I^{1} \cap I^{2}$.

Serial rules satisfy demand monotonicity because the revenue collected by charging any given price cannot decrease when the economy becomes richer: see Figure 6.1. As a consequence, no serial price increases, guaranteeing that no agent whose preferences are fixed can be worse off, even if the preferences in the richer economy justify that one or more additional units of the good be produced.

Our first result combines demand monotonicity with efficiency. Recall from Example 2 that restrictions are needed to guarantee efficiency of the serial allocations. We focus on $\mathcal{L}^{1}$, the domain of all economies satisfying Assumption L1. 


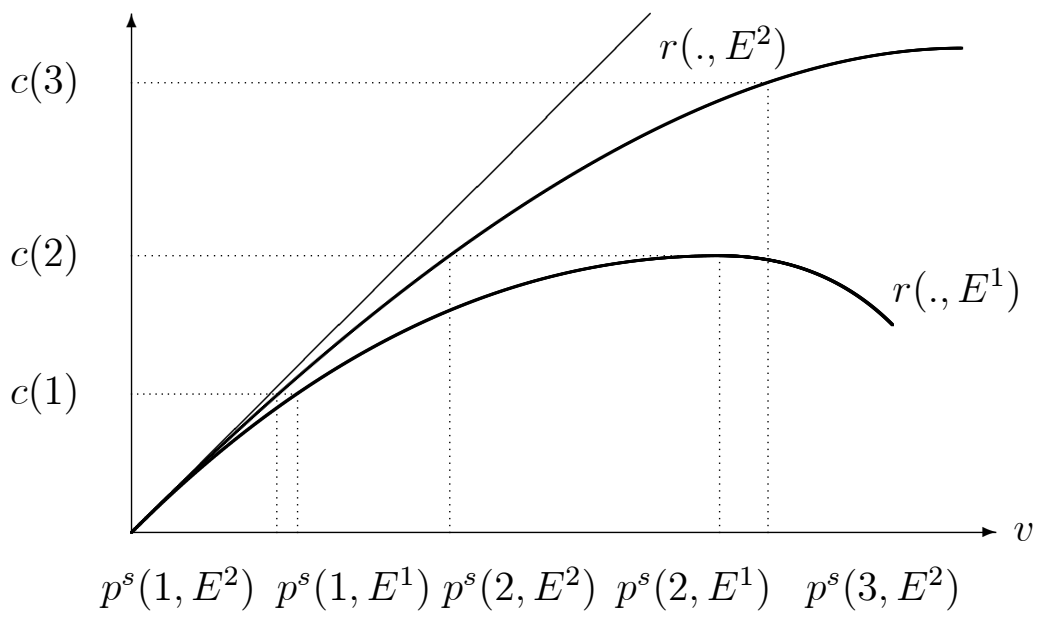

Figure 6.1: Serial rules satisfy Demand Monotonicity

Theorem 3. An allocation rule on $\mathcal{L}^{1}$ satisfies efficiency and demand monotonicity if and only if it is serial.

Here is an intuition for this result. At any serial allocation, the incremental cost of each unit of the public good is borne precisely by the consumers of that unit. Loosely speaking, this guarantees a two-sided no-subsidization property: the poorer (lower-valuation) agents do not subsidize the consumption of the richer, and the richer do not subsidize the poorer either.

To see why the poorer cannot subsidize the richer, suppose they do. Imagine a negative shift in the distribution of the richer agents' valuations. If this movement is large enough, efficiency forces a reduction in production down to the maximal consumption level of the poorer. The poorer agents can only benefit from the corresponding decrease in total cost, a contradiction to demand monotonicity.

The reason why the richer agents cannot subsidize the poorer is more subtle. Consider the largest consumers of the public good - those consuming up to the very last unit that is produced. Suppose first that the maximal revenue that can be collected in the economy coincides with the incremental cost of that last unit. In that particular case, efficiency forces us to produce that unit (condition 2 in Theorem 2) and the agents consuming it cannot pay a price larger than the one just covering the incremental cost (condition 3 in Theorem 2). Next, imagine a 
positive shift in the distribution of the valuations of the largest consumers of the good. Can we now charge them more than what is needed to cover the production cost of the last unit? The answer is negative: as long as the valuations of some agents among the largest consumers remain unchanged, asking them to pay more contradicts demand monotonicity. Consequently, the price of the last unit cannot exceed the serial price in this case either. An induction argument shows that the reasoning applies to the other produced units as well, and feasibility completes the argument.

Next we turn to population monotonicity. The axiom states that an increase in population should not be detrimental to anyone. To the extent that all agents are affected in an equal direction, this is an egalitarian-oriented axiom. The specific requirement that they gain is natural because the good is public; it is also appealing from an efficiency viewpoint as it makes it easier to accept new agents, thereby reducing average cost.

A slight change in the model is needed to discuss this property. An economy $(I, E)$ now consists of an interval of valuations $I=[\underline{v}, \bar{v}] \subset \mathbb{R}_{+}$and a function $E: \mathbb{R}_{+} \rightarrow[0, m(E)]$, where the positive real number $m(E)$ measures population size. We require $E$ to be continuous, nondecreasing, and such that $E(\underline{v})=0<$ $E(v)<m(E)=E(\bar{v})$ whenever $\underline{v}<v<\bar{v}$. Note that $E$ need not be strictly increasing on $I$ : this guarantees that $\left(c o\left(I^{1} \cup I^{2}\right), E^{1}+E^{2}\right)$ (where co denotes the convex hull) is a well-defined economy whenever $\left(I^{1}, E^{1}\right),\left(I^{2}, E^{2}\right)$ are. Let $\overline{\mathcal{L}}$ denote the set of such linear economies of all sizes and let $\overline{\mathcal{D}} \subseteq \overline{\mathcal{L}}$. Allocation rules are redefined on the domain $\overline{\mathcal{D}}$ in the obvious way.

Population monotonicity. If $\left(I^{1}, E^{1}\right),\left(I^{2}, E^{2}\right) \in \overline{\mathcal{D}}, \varphi\left(I^{1}, E^{1}\right)=\left(\mathbf{x}^{1}, \mathbf{y}^{1}\right)$ and $\varphi\left(c o\left(I^{1} \cup I^{2}\right), E^{1}+E^{2}\right)=\left(\mathbf{x}^{12}, \mathbf{y}^{12}\right)$, then $v \mathbf{y}^{12}(v)-\mathbf{x}^{12}(v) \geq v \mathbf{y}^{1}(v)-\mathbf{x}^{1}(v)$ for all $v \in I^{1}$.

Serial rules satisfy population monotonicity for essentially the same reason that they satisfy demand monotonicity. An increase in population raises the revenue that can be collected by charging any given price, which translates into a more favorable serial pricing scheme. No agent initially present can be made worse off by such a change, even if additional units are produced in the larger economy. See Figure 6.2.

Not every efficient population-monotonic rule is serial, however. In the example below, the production level is always the serial level and the pricing scheme 


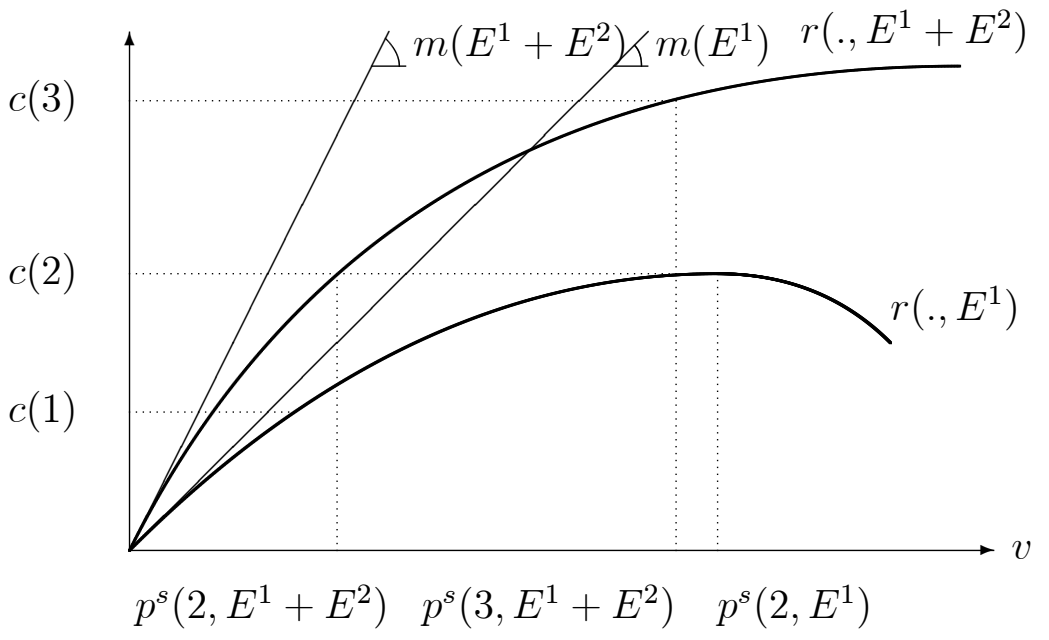

Figure 6.2: Serial rules satisfy Population Monotonicity

differs from the serial scheme only for the first two units. When at least two units are produced, the first unit is priced above the serial price and the second unit is priced below the serial price - exactly at the level ensuring budget balance.

Example 5. Let $\overline{\mathcal{D}}=\overline{\mathcal{L}}$, let $(I, E) \in \overline{\mathcal{D}}$ with $I=[\underline{v}, \bar{v}]$, and let $y^{s}$ be the serial production level for $(I, E)$. Define $\varphi(I, E)=(\mathbf{x}, \mathbf{y})$ as follows. If $y^{s}<2$, let $(\mathbf{x}, \mathbf{y})$ be a serial allocation. If $y^{s} \geq 2$, construct the nondecreasing pricing scheme $(0, p(., E))$ as follows. First, let $(J, F)$ be the sub-economy of $(I, E)$ obtained by removing all agents whose valuation exceeds the threshold $\widehat{v}$, where $\widehat{v}$ is chosen such that the maximal revenue in $(J, F)$ is exactly $C(2)$. Thus, $J=[\underline{v}, \widehat{v}], F(v)=$ $\min \{E(v), E(\widehat{v})\}$ for all $v \in \mathbb{R}_{+}$, and $\max _{v \in \mathbb{R}_{+}} r(v, F)=C(2)$. Let

$$
p(1, E)=\min \left\{v^{s}(1, F), b(E)\right\},
$$

where $v^{s}(1, F)$ is the smallest valuation $v \in \mathbb{R}_{+}$such that $r(v, F) \geq c(1)$ and $b(E)$ is the smallest valuation such that $r(b(E), E) \geq \frac{C(1)+C(2)}{2}$. Choose $p(2, E)$ to guarantee that $r(p(1, E), E)+r(p(2, E), E)=C(1)+C(2)$, and let $p(y, E)=$ $v^{s}(y, E)$ for all $y \geq 3$, where $v^{s}(y, E)$ is the smallest valuation $v \in \mathbb{R}_{+}$such that $r(v, E) \geq c(y)$. Let $(\mathbf{x}, \mathbf{y})$ be any allocation generated by this pricing scheme $(0, p(., E))$ and such that $\bar{y}=y^{s}$. One can show that the rule $\varphi$ thus defined is population-monotonic. 
A popular variant of our model is the so-called binary model, where production of the public good is restricted to be 1 or 0 : a public project can be undertaken or rejected. If we allow the cost function $C$ to take infinite values, the binary model is a particular case of of our model with $c(y)=+\infty$ for $y \geq 2$. In this much simplified environment, linearity of preferences is no longer a restriction since only the valuation of the first unit matters. Clearly, the rule just constructed cannot be distinguished from a serial rule in this context. In fact, one can show that the serial rules are the only efficient population-monotonic rules.

Theorem 4. In the binary model, an allocation rule on $\overline{\mathcal{L}}$ satisfies efficiency and population monotonicity if and only if it is serial.

The intuition is simpler than for Theorem 3. Efficiency rules out any production when population is small enough. It follows that no agent (in particular, no low-valuation agent) should be worse off than when consuming and paying nothing: otherwise, they would gain from a sufficiently large decrease in population, contradicting population monotonicity. As a result, when efficiency requires to produce one unit but the average cost is sufficiently large, low-valuation agents must be excluded and cannot pay a positive cost share.

We are close to the definition of serial allocations, except that the excluded agents could possibly be assigned a negative cost share. If they do, imagine they all leave the economy. Some of the remaining agents would necessarily gain from this departure, violating population monotonicity.

\section{Generalization to ordered quasi-linear preferences}

Our objective in this section is to generalize the results presented in the previous sections to the domain of quasi-linear preferences.

There is a set $\mathcal{T}$ of possible types. For each $t \in \mathcal{T}$ the preferences of (an agent of) type $t$ are represented by a valuation function $V(., t): \mathbb{N} \rightarrow \mathbb{R}_{+}$that satisfies $V(0, t)=0$, is nondecreasing, and is concave: defining $v(y, t)=V(y, t)-V(y-1, t)$ for every $y \in \mathbb{N} \backslash\{0\}, v(y, t) \geq v(y+1, t)$ for all $y \in \mathbb{N} \backslash\{0\}$. Let $\mathcal{V}$ denote the set of such valuation functions. The number $V(y, t)$ is the total contribution that an agent of type $t$ is willing to make to consume $y$ units of the public good; $V(y, t)-x$ measures her welfare from the bundle $(y, x)$.

We are not able to characterize incentive-compatible allocations at that level of generality. Because the marginal valuations $v(y, t)$ cannot be ordered as a function

of type only, an agent of type $t$ may end up consuming more or less than an agent 
of type $t^{\prime}>t$, depending on the distribution of types and the cost function. This is a well known difficulty. The classical solution, initiated by Mirrlees (1971) and Spence (1973), consists of introducing the following assumption on the mapping $V: \mathbb{R}_{+} \rightarrow \mathcal{V}$.

Single-crossing property. For all $t, t^{\prime} \in \mathcal{T}$ and all $y, y^{\prime} \in \mathbb{N} \backslash\{0\}, v(y, t) \leq$ $v\left(y, t^{\prime}\right) \Leftrightarrow v\left(y^{\prime}, t\right) \leq v\left(y^{\prime}, t^{\prime}\right)$.

A crucial consequence of this assumption is that, like in the linear case, agents are naturally ordered according to the "level of intensity" of their preference for the public good: we can assume that $\mathcal{T}=\mathbb{R}_{+}$and, for all $t, t^{\prime} \in \mathbb{R}_{+}, t<t^{\prime} \Leftrightarrow$ $v(y, t)<v\left(y, t^{\prime}\right) \forall y \in \mathbb{N} \backslash\{0\}$.

We add an assumption on $V$ guaranteeing that there is no gap in the support of these intensity levels. It corresponds to our ealier restriction that, in the linear case, the support of the distribution of valuations is connected.

Continuity. For all $t \in \mathbb{R}_{+}$and all $\varepsilon>0$, there exists $\delta>0$ such that $\sup _{y \in \mathbb{N}}\left|V(y, t)-V\left(y, t^{\prime}\right)\right|<\varepsilon$ for all $t^{\prime} \in \mathbb{R}_{+}$such that $\left|t-t^{\prime}\right|<\delta$.

Given $V$, a quasi-linear economy is a pair $(T, E)$ where $T=[\underline{t}, \bar{t}] \subset \mathbb{R}_{+}$is a nondegenerate interval of types and $E: \mathbb{R}_{+} \rightarrow[0,1]$ is continuous, strictly increasing on $T$, and such that $E(\underline{t})=0$ and $E(\bar{t})=1$. For any $t \in T, E(t)$ represents the proportion of types whose valuation function is $V\left(., t^{\prime}\right)$ for some $t^{\prime} \leq t$. Because of the single-crossing property and continuity, a quasi-linear economy $(T, E)$ shares the most important features of a linear economy of the type described in Section 2 .

An allocation for $(T, E)$ is a pair $(\mathbf{x}, \mathbf{y})$, where $\mathbf{x}: T \rightarrow \mathbb{R}$ is an integrable mapping specifying the cost share of every type of agent and $\mathbf{y}: T \rightarrow \mathbb{N}$ specifies the level of consumption of the good. This allocation is incentive-compatible if

$$
V(\mathbf{y}(t), t)-\mathbf{x}(t) \geq V\left(\mathbf{y}\left(t^{\prime}\right), t\right)-\mathbf{x}\left(t^{\prime}\right) \text { for all } t, t^{\prime} \in T
$$

and it is feasible if $\int_{T} \mathbf{x} d E \geq C\left(\sup _{t \in T} \mathbf{y}(t)\right)$.

A pricing scheme for $(T, E)$ is a pair $(a, p)$ as before, with $p(y) \geqslant v(y, \bar{t})$ for all $y$ sufficiently large. We say that a pricing scheme $(a, p)$ is standard for $(T, E)$ if $v(y, \underline{t}) \leq p(y) \leq v(y, \bar{t})$ for all $y \in \mathbb{N} \backslash\{0\}$. The type scheme generated by $(a, p)$ is the function $\tau: \mathbb{N} \backslash\{0\} \rightarrow \mathbb{R}_{+}$defined by $\tau(y)=t \Leftrightarrow v(y, t)=p(y)$. It identifies for each unit $y$ the type $\tau(y)$ whose valuation of the $y$ th unit is exactly $p(y)$, that is, the type just indifferent between buying the $y$ th unit or not. We call $\tau$ nondecreasing if and only if $\tau(y) \leq \tau(y+1)$ for all $y \in \mathbb{N} \backslash\{0\}$. 
A nondecreasing type scheme may well be generated by a standard pricing scheme for $(T, E)$ that itself fails to be nondecreasing: if $\tau(y)=\tau(y+1)=t$ for some unit $y$, then $p(y)=v(y, t)>v(y+1, t)=p(y+1)$.

\section{Theorem 5.}

(a) If $(a, p)$ is a standard pricing scheme for $(T, E)$ generating a nondecreasing type scheme, then any allocation $(\mathbf{x}, \mathbf{y})$ for $(T, E)$ satisfying

$$
\mathbf{y}(t) \in\{y \in \mathbb{N} \mid p(y) \leq v(y, t) \text { and } v(y+1, t) \leq p(y+1)\}
$$

and

$$
\mathbf{x}(t)=a+\sum_{y=1}^{\mathbf{y}(t)} p(y)
$$

for all $t \in T$ is incentive-compatible.

(b) If $(\mathbf{x}, \mathbf{y})$ is an incentive-compatible allocation for $(T, E)$, then there exists a unique standard pricing scheme $(a, p)$ for $(T, E)$ generating a nondecreasing type scheme and satisfying (7.2) and (7.3).

The proof of the above theorem parallels the proof of Theorem 1 and is therefore omitted.

The definition of serial allocations is straightforward. For each $y \in \mathbb{N} \backslash\{0\}$, define $E_{y}: \mathbb{R}_{+} \rightarrow[0,1]$ by

$$
E_{y}(w)=E\left(\sup \left\{t \in \mathbb{R}_{+} \mid v(y, t) \leq w\right\}\right) .
$$

Thus, $E_{y}(w)$ is the proportion of types whose valuation of the $y$ th unit does not exceed $w$. Observe that $E_{y}$ is a distribution of valuations, whereas $E$ is a distribution of types. When preferences are linear, $E_{y}=E_{y^{\prime}}$ for all $y, y^{\prime}$. Define the revenue function $r_{y}$ for unit $y$ by $r_{y}(w)=w\left(1-E_{y}(w)\right)$ for all $w \in \mathbb{R}_{+}$and redefine $Y^{s}$ to be the set of all $y \in \mathbb{N}$ such that

$$
r_{y}(w) \geq c(y)
$$

for some $w \in \mathbb{R}_{+}$. Write $Y^{s}=\left\{0, \ldots, y^{s}\right\}$ and let $v^{s}(y)$ be the smallest $w \in \mathbb{R}_{+}$ satisfying (7.4). Extend the mapping $v^{s}$ by setting $v^{s}(y)=v(y, \overline{\bar{t}})$ for all $y>y^{s}$, where $\overline{\bar{t}}$ is an arbitrary type larger than $\bar{t}$. The serial allocations for $(T, E)$ are the allocations that are Pareto-equivalent to those generated by the pricing scheme $\left(0, v^{s}\right)$ and have the production level $y^{s}$. Note that the serial pricing scheme is nondecreasing. 
As before, we call a feasible incentive-compatible allocation efficient if it is not Pareto-dominated by any feasible incentive-compatible allocation. Strict concavity of the revenue functions $r_{y}$, for all $y \in \mathbb{N} \backslash\{0\}$, is not sufficient to guarantee efficiency of the serial allocations, as illustrated in the following example.

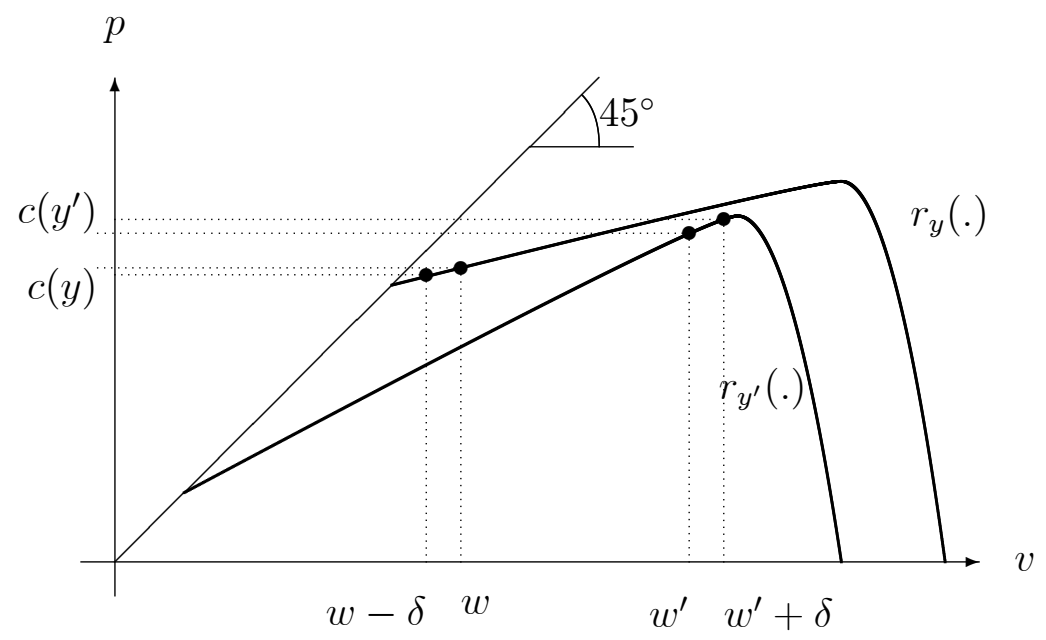

Figure 7.1: Strict concavity of the revenue functions does not guarantee efficiency of serial allocations

Example 6. Let $(T, E)$ be such that for some $y, y^{\prime} \in \mathbb{N} \backslash\{0\}, y<y^{\prime}$, the strictly concave revenue functions are such as drawn in Figure 7.1. The serial allocations are generated by a pricing scheme charging $w$ for the $y$ th unit and $w^{\prime}$ for the $y^{\prime}$ th unit. Decreasing the price of the $y$ th unit and increasing that of the $y^{\prime}$ th unit by an amount $\delta$ increases total revenue. Serial allocations are not efficient.

We therefore introduce the following restriction.

Assumption QL1. Let $y, y^{\prime} \in \mathbb{N} \backslash\{0\}$ be such that $y \leq y^{\prime}$, let $\gamma, \gamma^{\prime} \in \mathbb{R}_{++}$be such that $\gamma<\gamma^{\prime} \leq \max _{w \in \mathbb{R}_{+}} r_{y^{\prime}}(w)$, and let $w, w^{\prime}$ be the smallest valuations in $\mathbb{R}_{+}$such that $r_{y}(w)=\gamma$ and $r_{y^{\prime}}\left(w^{\prime}\right)=\gamma^{\prime}$, respectively. There exists $\varepsilon>0$ such that $r_{y^{\prime}}\left(w^{\prime}+\delta\right)-r_{y^{\prime}}\left(w^{\prime}\right)<r_{y}(w)-r_{y}(w-\delta)$ whenever $0<\delta<\varepsilon$. 
This assumption reduces to Assumption L1 in the linear case because the revenue functions for the different units all coincide. Let $\mathcal{Q} \mathcal{L}^{1}$ denote the set of quasi-linear economies $(T, E)$ satisfying Assumption QL1.

An (allocation) rule on $\mathcal{Q} \mathcal{L}^{1}$ is a mapping $\varphi$ assigning to each $(T, E) \in \mathcal{Q} \mathcal{L}^{1}$ an incentive-compatible and feasible allocation for $(T, E)$. A serial rule selects a serial allocation in every economy. The efficiency axiom is adapted in the obvious way. The definition of demand monotonicity is also clear. If $\left(T^{1}, E^{1}\right),\left(T^{2}, E^{2}\right) \in \mathcal{Q} \mathcal{L}^{1}$, write $\left(T^{1}, E^{1}\right) \unlhd\left(T^{2}, E^{2}\right)$ if and only if $E^{2}(t) \leq E^{1}(t)$ for all $t \in \mathbb{R}_{+}$. This implies that for every $y \in \mathbb{N} \backslash\{0\}, E_{y}^{2}(w) \leq E_{y}^{1}(w)$ for all $w \in \mathbb{R}_{+}$: for each $y$ and $w$, the proportion of types whose valuation of the $y$ th unit exceeds $w$ is larger in $\left(T^{2}, E^{2}\right)$ than in $\left(T^{1}, E^{1}\right)$. Demand monotonicity requires that if $\left(T^{1}, E^{1}\right) \unlhd$ $\left(T^{2}, E^{2}\right), \varphi\left(T^{1}, E^{1}\right)=\left(\mathbf{x}^{1}, \mathbf{y}^{1}\right)$ and $\varphi\left(T^{2}, E^{2}\right)=\left(\mathbf{x}^{2}, \mathbf{y}^{2}\right)$, then $V\left(\mathbf{y}^{2}(t), t\right)-\mathbf{x}^{2}(t) \geq$ $V\left(\mathbf{y}^{1}(t), t\right)-\mathbf{x}^{1}(t)$ for all $t \in T^{1} \cap T^{2}$.

Theorem 6. An allocation rule on $\mathcal{Q} \mathcal{L}^{1}$ satisfies efficiency and demand monotonicity if and only if it is serial.

\section{Conclusion}

We have provided an axiomatic justification for the serial solution to the problem of provision and cost-sharing of an excludable public good under incentive constraints. Our defense combines the requirement of incentive-constrained efficiency with considerations of fairness as expressed by the demand and population monotonicity axioms. An interesting step in establishing our characterization result for linear preferences (Theorem 3) consists in an explicit description of the incentive-constrained efficient allocations (Theorem 2): the four properties in that theorem are particularly easy to check.

Our entire analysis relies on two crucial assumptions. First, agents are ranked along a single dimension measuring the intensity of their preference for the public good: if an agent is willing to pay more than another for the first unit of the good, then so is she for all other units. On the one hand we find this assumption plausible; on the other hand, dropping it would make the problem immensely more difficult.

The second assumption is that of a single, homogenous, public good. Our main defense of the serial solution (Theorems 3 and 6) generalizes easily to the case of several public goods if the cost function is additively separable and the preferences are separable and sufficiently diverse. In that case, any efficient and 
demand-monotonic rule must be serial in each good. Let us briefly explain this point in the linear case.

Assume that there is a set $L$ of excludable public goods. It costs $C(y)=$ $\sum_{l \in L} c_{l}\left(y_{l}\right)$ units of money to produce the bundle of public goods $y \in \mathbb{N}^{L}$. A preference is represented by a vector of valuations $v \in \mathbb{R}_{+}^{L}$, implying that the valuation of good $l$ does not depend on the consumption of good $l^{\prime}$. An economy is a pair $(I, E)$ where $I=\prod_{l \in L}\left[\underline{v}_{l}, \bar{v}_{l}\right] \subset \mathbb{R}_{+}^{L}$ is the product of non-degenerate intervals of valuations and $E: \mathbb{R}_{+}^{L} \rightarrow[0,1]$ is a multivariate, continuous, strictly increasing distribution function on $I$. Allocations are defined in the obvious way. Writing $\left(I^{1}, E^{1}\right) \unlhd\left(I^{2}, E^{2}\right)$ if and only if $E^{2}(v) \leq E^{1}(v)$ for all $v \in \mathbb{R}_{+}^{L}$, demand monotonicity requires that if $\left(I^{1}, E^{1}\right) \unlhd\left(I^{2}, E^{2}\right), \varphi\left(I^{1}, E^{1}\right)=\left(\mathbf{x}^{1}, \mathbf{y}^{1}\right)$ and $\varphi\left(I^{2}, E^{2}\right)=\left(\mathbf{x}^{2}, \mathbf{y}^{2}\right)$, then $v \mathbf{y}^{2}(v)-\mathbf{x}^{2}(v) \geq v \mathbf{y}^{1}(v)-\mathbf{x}^{1}(v)$ for all $v \in I^{1} \cap I^{2}$, where $v \mathbf{y}^{1}$ and $v \mathbf{y}^{2}$ are now inner products.

If cross-subsidization is forbidden, that is, the revenue collected from consumers of good $l$ cannot be used to finance the production of good $l^{\prime}$, then Theorem 3 implies that any efficient and demand-monotonic rule must be serial. But the following simple argument shows that demand monotonicity does forbid crosssubsidization. Suppose that in some economy, the production of good $l$ is crosssubsidized by the consumers of good $l^{\prime}$. Consider a negative shift in the marginal distribution of valuations of good $l$ large enough that efficiency requires to stop producing this good (the marginal distributions of valuations of all other goods remaining unchanged). This is goods news for the consumers of good $l^{\prime}$, who stop cross-subsidizing good $l$. In particular, any agent who was consuming good $l^{\prime}$ but not good $l$ benefits strictly from the change, contradicting demand monotonicity. The argument requires only a sufficient degree of preference diversity: there must exist an agent with an arbitrarily high valuation of one good and an arbitrary low valuation of the other.

Even though the incentive-constrained axiomatic approach followed here produces interesting results, we believe it is unlikely to be equally successful in other contexts. In the environment we have studied, incentive-constrained efficiency turns out to be compatible with powerful monotonicity conditions expressing natural fairness desiderata. This is largely due to the fact that the produced good is a public one. In private-goods problems, such as the design of fair incentivecompatible collective insurance schemes, it may be much harder to formulate fairness requirements that can be successfully combined with incentive-constrained efficiency. 


\section{Appendix}

\subsection{Proof of Theorem 1}

Let $(I, E)$ be an economy.

Statement (a). Let $(a, p)$ be a nondecreasing pricing scheme for $(I, E)$ and fix $v, v^{\prime} \in I$. By (3.2) and (3.3), $v \mathbf{y}(v)-\mathbf{x}(v)=v \mathbf{y}(v)-\left(a+\sum_{q=1}^{\mathbf{y}(v)} p(q)\right) \geq v y-$ $\left(a+\sum_{q=1}^{y} p(q)\right)$ for all $y \in \mathbb{N}$. Taking $y=\mathbf{y}\left(v^{\prime}\right), v \mathbf{y}(v)-\mathbf{x}(v) \geq v \mathbf{y}\left(v^{\prime}\right)-(a+$ $\left.\sum_{q=1}^{\mathbf{y}\left(v^{\prime}\right)} p(q)\right)=v \mathbf{y}\left(v^{\prime}\right)-\mathbf{x}\left(v^{\prime}\right)$.

Statement (b). Let $(\mathbf{x}, \mathbf{y})$ be an incentive-compatible allocation for $(I, E)$. For any $v, v^{\prime} \in I$, applying $(3.1)$ to both $\left(v, v^{\prime}\right)$ and $\left(v^{\prime}, v\right)$ gives

$$
v\left(\mathbf{y}\left(v^{\prime}\right)-\mathbf{y}(v)\right) \leq \mathbf{x}\left(v^{\prime}\right)-\mathbf{x}(v) \leq v^{\prime}\left(\mathbf{y}\left(v^{\prime}\right)-\mathbf{y}(v)\right) .
$$

It follows that both $\mathbf{y}$ and $\mathbf{x}$ are non-decreasing functions and $\mathbf{x}(v)=\mathbf{x}\left(v^{\prime}\right)$ if and only if $\mathbf{y}(v)=\mathbf{y}\left(v^{\prime}\right)$. Since $I$ is compact, both $\mathbf{x}$ and $\mathbf{y}$ must have a finite range: there exists $N \in \mathbb{N}$ such that

$$
\begin{aligned}
& \mathbf{y}(I)=\left\{y^{0}, \ldots, y^{N}\right\}, \\
& \mathbf{x}(I)=\left\{x^{0}, \ldots, x^{N}\right\},
\end{aligned}
$$

with $y^{n}<y^{n+1}$ and $x^{n}<x^{n+1}$ whenever $n, n+1 \in\{0, \ldots, N\}$, and $\mathbf{x}(v)=x^{n}$ if and only if $\mathbf{y}(v)=y^{n}$.

For $n=0, \ldots, N$, define $I^{n}=\left\{v \in I \mid(\mathbf{x}(v), \mathbf{y}(v))=\left(x^{n}, y^{n}\right)\right\}$ and $v^{n}=\inf I^{n}$. Note that $\underline{v}=v^{0} \leq \ldots \leq v^{N} \leq \bar{v}$. Moreover, because $E$ is strictly increasing, $v^{n}=\sup I^{\bar{n}-1}$ for $n=1, \ldots, N$. Applying (9.1) to $v \in I^{n-1}, v^{\prime} \in I^{n}$ and taking limits as $v$ tends to $\sup I^{n-1}$ and $v^{\prime}$ tends to inf $I^{n}$,

$$
\sup I^{n-1} \leq \frac{x^{n}-x^{n-1}}{y^{n}-y^{n-1}} \leq \inf I^{n}
$$

that is,

$$
v^{n}=\frac{x^{n}-x^{n-1}}{y^{n}-y^{n-1}}
$$

for $n=1, \ldots, N$.

Define now the standard pricing scheme $(a, p)$ as follows: $p(y)=v^{\min \left\{n \mid y \leq y^{n}\right\}}$ if $1 \leq y \leq y^{N}, p(y)=\bar{v}$ if $y>y^{N}$ otherwise, and $a=x^{0}-\underline{v} y^{0}$. By construction, $(a, p)$ satisfies equations (3.2) and (3.3) for every $v \in I$. 
It remains to be shown that no other standard pricing scheme does. Suppose $\left(a^{\prime}, p^{\prime}\right)$ is a standard scheme satisfying

$$
\mathbf{y}(v) \in\left\{y \in \mathbb{N} \mid p^{\prime}(y) \leq v \leq p^{\prime}(y+1)\right\}
$$

and

$$
\mathbf{x}(v)=a^{\prime}+\sum_{y=1}^{\mathbf{y}(v)} p^{\prime}(y)
$$

for all $v \in I$. We claim that $\left(a^{\prime}, p^{\prime}\right)=(a, p)$.

First, let us show that

$$
a^{\prime}=a \text { and } p^{\prime}(y)=p(y) \text { if } 1 \leq y \leq y^{0} .
$$

Applying (3.3), (9.4) with any $v$ such that $\mathbf{y}(v)=y^{0}$, we get $a+\sum_{y=1}^{y^{0}} p(y)=$ $a^{\prime}+\sum_{y=1}^{y^{0}} p^{\prime}(y)$. If $y^{0}=0$, we immediately get $a=a^{\prime}$, hence (9.5). If $y^{0}>0$, then $a^{\prime}=a+\sum_{y=1}^{y^{0}}\left(p(y)-p^{\prime}(y)\right)=a+\sum_{y=1}^{y^{0}}\left(\underline{v}-p^{\prime}(y)\right)$. Apply (9.3) with $v=\underline{v}$. Since $\mathbf{y}(\underline{v})=y^{0}$, we get $p^{\prime}\left(y^{0}\right) \leq \underline{v}$. But $p^{\prime}$ is nondecreasing and takes values in $I$, hence $p^{\prime}(y)=\underline{v}$ for $y=1, \ldots, y^{0}$ and $a^{\prime}=a$, proving (9.5).

Next, let us show that

$$
p^{\prime}(y)=p(y) \text { if } y^{0}+1 \leq y \leq y^{1} .
$$

Apply (3.3), (9.4) with any $v$ such that $\mathbf{y}(v)=y^{1}$. Taking (9.5) into account, $\sum_{y=y^{0}+1}^{y^{1}} p(y)=\sum_{y=y^{0}+1}^{y^{1}} p^{\prime}(y)$. If $y^{1}=y^{0}+1,(9.6)$ follows at once. If $y^{1}>y^{0}+1$, suppose (9.6) fails. Since $p^{\prime}$ is nondecreasing, we must have $p^{\prime}\left(y^{0}+1\right)<p\left(y^{0}+1\right)=$ $v^{1}=p\left(y^{1}\right)<p^{\prime}\left(y^{1}\right)$. Apply (9.3) with $v=v^{1}$. By construction, $\mathbf{y}\left(v^{1}\right) \in\left\{y^{0}, y^{1}\right\}$. If $\mathbf{y}\left(v^{1}\right)=y^{0},(9.3)$ implies $v^{1} \leq p^{\prime}\left(y^{0}+1\right)$, a contradiction. If $\mathbf{y}\left(v^{1}\right)=y^{1}$, (9.3) implies $p^{\prime}\left(y^{1}\right) \leq v^{1}$, again a contradiction. Thus $p^{\prime}(y)=v^{1}=p(y)$ for $y=y^{0}+1, \ldots, y^{1}$.

Continuing in this way, we obtain $p^{\prime}(y)=p(y)$ if $y^{n-1}+1 \leq y \leq y^{n}$ for $n=1, \ldots N$. Since $p^{\prime}$ takes values in $I$, it is clear that $p^{\prime}(y)=\bar{v}$ for $y>y^{N}$. Hence $\left(a^{\prime}, p^{\prime}\right)=(a, p)$.

\subsection{Proof of Lemma 1}

Let $(I, E)$ be an economy. Given a standard pricing scheme $(a, p)$ for $(I, E)$, define

$$
P(y)=a+\sum_{q=1}^{y} p(q)
$$


for all $y \in \mathbb{N}$. We call $P$ a standard contribution scheme. It is convex, that is, $P(y)-P(y-1) \leq P(y+1)-P(y)$ for all $y \in \mathbb{N} \backslash\{0\}$, because the mapping $p$ is nondecreasing. If the above inequality is strict, we call $y$ a kink of $P$ and denote by $K(P)$ the set of kinks of $P$. Note that $K(P) \subseteq\{y, \ldots, \bar{y}\}$ because $p(y)=\underline{v}$ for all $y \leq y$ and $p(y)=\bar{v}$ for all $y>\bar{y}$.

Now, let $(\mathbf{x}, \mathbf{y}),\left(\mathbf{x}^{\prime}, \mathbf{y}^{\prime}\right) \in A(I, E)$ and let $P, P^{\prime}$ be their representing standard contribution schemes. It follows from Theorem 1 that for all $v \in I$,

$$
v \mathbf{y}(v)-\mathbf{x}(v) \geq v y-P(y) \text { for all } y \in \mathbb{N},
$$

and

$$
v \mathbf{y}^{\prime}(v)-\mathbf{x}^{\prime}(v) \geq v y-P^{\prime}(y) \text { for all } y \in \mathbb{N} \text {. }
$$

Step 1. If $P^{\prime}<P$, then $\left(\mathbf{x}^{\prime}, \mathbf{y}^{\prime}\right)$ Pareto-dominates $(\mathbf{x}, \mathbf{y})$.

Suppose $P^{\prime}<P$, that is, $P^{\prime}(y) \leq P(y)$ for all $y \in \mathbb{N}$ and $P^{\prime}(y)<P(y)$ for some $y \in \mathbb{N}$. By convexity of $P$, at least one such strict inequality must hold for some $y \in K\left(P^{\prime}\right)$. For all $v \in I$,

$$
\begin{aligned}
v \mathbf{y}^{\prime}(v)-\mathbf{x}^{\prime}(v) & \geq v \mathbf{y}(v)-P^{\prime}(\mathbf{y}(v)) \\
& \geq v \mathbf{y}(v)-P(\mathbf{y}(v)) \\
& =v \mathbf{y}(v)-\mathbf{x}(v) .
\end{aligned}
$$

Next, pick $y \in K\left(P^{\prime}\right)$ such that $P^{\prime}(y)<P(y)$. Define $v_{+}=P^{\prime}(y+1)-P^{\prime}(y)$ and $v_{-}=P^{\prime}(y)-P^{\prime}(y-1)$. For all $v \in\left(v_{-}, v_{+}\right)$,

$$
\begin{aligned}
v \mathbf{y}^{\prime}(v)-\mathbf{x}^{\prime}(v) & =v y-P^{\prime}(y) \\
& >v y^{\prime}-P\left(y^{\prime}\right)
\end{aligned}
$$

for all $y^{\prime} \in \mathbb{N}$ and, in particular, $v \mathbf{y}^{\prime}(v)-\mathbf{x}^{\prime}(v)>v \mathbf{y}(v)-\mathbf{x}(v)$. Since $\left(v_{-}, v_{+}\right)$is an $E$-positive subset of $I,\left(\mathbf{x}^{\prime}, \mathbf{y}^{\prime}\right)$ Pareto-dominates $(\mathbf{x}, \mathbf{y})$.

Step 2. If $\left(\mathbf{x}^{\prime}, \mathbf{y}^{\prime}\right)$ Pareto-dominates $(\mathbf{x}, \mathbf{y})$, then $P^{\prime}<P$.

Suppose $\left(\mathbf{x}^{\prime}, \mathbf{y}^{\prime}\right)$ Pareto-dominates $(\mathbf{x}, \mathbf{y})$. Suppose, by way of contradiction, that $P^{\prime}(y)>P(y)$ for some $y \in \mathbb{N}$.

If $y \in K\left(P^{\prime}\right)$, we obtain an immediate contradiction since for all $v \in\left(P^{\prime}(y)-\right.$ $\left.P^{\prime}(y-1), P^{\prime}(y+1)-P^{\prime}(y)\right)$,

$$
\begin{aligned}
v \mathbf{y}(v)-\mathbf{x}(v) & \geq v y-P(y) \\
& >v y-P^{\prime}(y) \\
& =v \mathbf{y}^{\prime}(v)-\mathbf{x}^{\prime}(v) .
\end{aligned}
$$


If $y \notin K\left(P^{\prime}\right)$, then $\left\{y^{\prime} \in K\left(P^{\prime}\right) \mid y^{\prime} \leq y\right\} \neq \varnothing$ or $\left\{y^{\prime} \in K\left(P^{\prime}\right) \mid y^{\prime} \geq y\right\} \neq \varnothing$. Let us focus on the first case; a similar argument applies in the other case. Define $y^{0}=\max \left\{y^{\prime} \in K\left(P^{\prime}\right) \mid y^{\prime} \leq y\right\}$. Then for all $v \in\left(\frac{P(y)-P^{\prime}\left(y^{0}\right)}{y-y^{0}}, \frac{P^{\prime}(y)-P^{\prime}\left(y^{0}\right)}{y-y^{0}}\right) \cap I$,

$$
\begin{aligned}
v \mathbf{y}(v)-\mathbf{x}(v) & >v y^{0}-P^{\prime}\left(y^{0}\right) \\
& \geq v \mathbf{y}^{\prime}(v)-P^{\prime}\left(\mathbf{y}^{\prime}(v)\right) \\
& =v \mathbf{y}^{\prime}(v)-\mathbf{x}^{\prime}(v),
\end{aligned}
$$

a contradiction.

\subsection{Proof of Lemma 2}

Let $(I, E)$ satisfy Assumption 1 and let $(a, p),\left(a^{\prime}, p^{\prime}\right)$ be nondecreasing pricing schemes for $(I, E)$ such that $\left(a^{\prime}, p^{\prime}\right) \preceq(a, p)$. Since $(I, E)$ is fixed, we write $r$ instead of $r(., E)$. Fix $\bar{y} \in \mathbb{N} \backslash\{0\}$ such that $p(\bar{y}) \leq v^{*}$. For any nondecreasing pricing scheme $\left(a^{\prime \prime}, p^{\prime \prime}\right)$, write

$$
R\left(a^{\prime \prime}, p^{\prime \prime}, \bar{y}\right)=a^{\prime \prime}+\sum_{y=1}^{\bar{y}} r\left(p^{\prime \prime}(y)\right) .
$$

We must prove that $R\left(a^{\prime}, p^{\prime}, \bar{y}\right) \leq R(a, p, \bar{y})$ and that $R\left(a^{\prime}, p^{\prime}, \bar{y}\right)<R(a, p, \bar{y})$ if $\left(a^{\prime}, p^{\prime}\right) \prec(a, p)$ and both schemes are standard.

First, observe that it is enough to prove these claims under the assumption that

$$
p^{\prime}(\bar{y}) \leq v^{*}
$$

Indeed, if this assumption is violated, define the nondecreasing pricing scheme $a^{\prime \prime}=a^{\prime}$ and $p^{\prime \prime}(y)=\min \left\{p^{\prime}(y), v^{*}\right\}$ if $y \leq \bar{y}, p^{\prime \prime}(y)=p^{\prime}(y)$ if $y>\bar{y}+1$. By construction, $\left(a^{\prime \prime}, p^{\prime \prime}\right) \prec\left(a^{\prime}, p^{\prime}\right), R\left(a^{\prime}, p^{\prime}, \bar{y}\right)<R\left(a^{\prime \prime}, p^{\prime \prime}, \bar{y}\right)$, and $p^{\prime \prime}(\bar{y}) \leq v^{*}$. Thus $R\left(a^{\prime}, p^{\prime}, \bar{y}\right)<R(a, p, \bar{y})$ if $R\left(a^{\prime \prime}, p^{\prime \prime}, \bar{y}\right) \leq R(a, p, \bar{y})$.

From now on, assume (9.10). We will use the following fact.

Theorem (Shorrocks (1983)). Let $p, p^{\prime} \in R_{+}^{K}$ be such that i) $p_{i} \leq p_{i+1}$ and $p_{i}^{\prime} \leq p_{i+1}^{\prime}$ for all $i=1, \ldots, K-1$, ii) $\sum_{i=1}^{k} p_{i}^{\prime} \leq \sum_{i=1}^{k} p_{i}$ for $k=1, \ldots, K$. If $g$ is a nondecreasing concave function, then $\sum_{i=1}^{K} g\left(p_{i}^{\prime}\right) \leq \sum_{i=1}^{K} g\left(p_{i}\right)$. This inequality is strict if $g$ is strictly concave and at least one of the inequalities in ii) is strict.

We distinguish three cases. 
Case 1. $a^{\prime}=a$ and $\sum_{y=1}^{\bar{y}} p^{\prime}(y)=\sum_{y=1}^{\bar{y}} p(y)$.

Since $p(y), p^{\prime}(y) \leq v^{*}$ for all $y=1, \ldots, \bar{y}$, Assumption 1 guarantees that $r$ is nondecreasing and concave on the desired range, namely, $\left[\min \left\{p(1), p^{\prime}(1)\right\}\right.$, $\left.\max \left\{p(\bar{y}), p^{\prime}(\bar{y})\right\}\right]$. If $(a, p),\left(a^{\prime}, p^{\prime}\right)$ are standard, then $p(y), p^{\prime}(y) \in\left[\underline{v}, v^{*}\right]$ for all $y=1, \ldots, \bar{y}$ and Assumption 1 also guarantees that $r$ is strictly concave on the relevant range. The result then follows directly from the Hardy, Littlewood and Pólya theorem.

Case 2. $a^{\prime}<a$ and $a^{\prime}+\sum_{y=1}^{\bar{y}} p^{\prime}(y)=a+\sum_{y=1}^{\bar{y}} p(y)$.

Let $\left(a^{\prime \prime}, p^{\prime \prime}\right)$ be the maximal nondecreasing pricing scheme such that $a^{\prime \prime}=a^{\prime}$ and $\left(a^{\prime \prime}, p^{\prime \prime}\right) \preceq(a, p)$. By Case $1, R\left(a^{\prime}, p^{\prime}, \bar{y}\right) \leq R\left(a^{\prime \prime}, p^{\prime \prime}, \bar{y}\right)$. Let $y^{*} \in\{1, \ldots, \bar{y}\}$ be such that $p^{\prime \prime}(y)>p(y)$ for all $y \leq y^{*}$ and $p^{\prime \prime}(y)=p(y)$ for all $y \geq y^{*}+1$. The key observation is that $r(v)-r\left(v^{\prime}\right) \leq v-v^{\prime}$ for all $v, v^{\prime} \in I$ such that $v^{\prime}<v$ (and this inequality is strict if $\underline{v}<v$ ). Therefore,

$$
\sum_{y=1}^{y^{*}}\left(r\left(p^{\prime \prime}(y)\right)-r(p(y))\right) \leq \sum_{y=1}^{y^{*}}\left(p^{\prime \prime}(y)-p(y)\right)=a-a^{\prime \prime} .
$$

Therefore $R\left(a^{\prime \prime}, p^{\prime \prime}, \bar{y}\right)=a^{\prime \prime}+\sum_{y=1}^{y^{*}} r\left(p^{\prime \prime}(y)\right)+\sum_{y=y^{*}+1}^{\bar{y}} r(p(y)) \leq a+\sum_{y=1}^{y^{*}} r(p(y))$ $+\sum_{y=y^{*}+1}^{\bar{y}} r(p(y))=R(a, p, \bar{y})$, hence $R\left(a^{\prime}, p^{\prime}, \bar{y}\right) \leq R(a, p, \bar{y})$. If $(a, p),\left(a^{\prime}, p^{\prime}\right)$ are standard and $\left(a^{\prime}, p^{\prime}\right) \prec(a, p)$, then inequality (9.11) is strict and $R\left(a^{\prime}, p^{\prime}, \bar{y}\right)<$ $R(a, p, \bar{y})$.

Case 3. $a^{\prime}<a$ and $a^{\prime}+\sum_{y=1}^{\bar{y}} p^{\prime}(y)<a+\sum_{y=1}^{\bar{y}} p(y)$.

Let $\left(a^{\prime \prime}, p^{\prime \prime}\right)$ be the maximal nondecreasing pricing scheme such that $a^{\prime \prime}=a$, $\left(a^{\prime \prime}, p^{\prime \prime}\right) \preceq(a, p)$ and $a^{\prime \prime}+\sum_{y=1}^{\bar{y}} p^{\prime \prime}(y)=a^{\prime}+\sum_{y=1}^{\bar{y}} p^{\prime}(y)$. By Case $2, R\left(a^{\prime}, p^{\prime}, \bar{y}\right) \leq$ $R\left(a^{\prime \prime}, p^{\prime \prime}, \bar{y}\right)$. Let $y^{*} \in\{1, \ldots, \bar{y}-1\}$ be such that $p^{\prime \prime}(y)=p(y)$ for all $y \leq y^{*}$ and $p^{\prime \prime}(y)<p(y)$ for all $y \geq y^{*}+1$. Given that $r$ is strictly concave on $\left[\underline{v}, v^{*}\right]$, it is strictly increasing on $\left[0, v^{*}\right]$. Since $p(\bar{y}) \leq v^{*}$, it follows that $r\left(p^{\prime \prime}(y)\right)<$ $r(p(y))$ for $y^{*}+1 \leq y \leq \bar{y}$. Consequently $R\left(a^{\prime \prime}, p^{\prime \prime}, \bar{y}\right)=a+\sum_{y=1}^{y^{*}} r(p(y))$ $+\sum_{y=y^{*}+1}^{\bar{y}} r\left(p^{\prime \prime}(y)\right)<a+\sum_{y=1}^{y^{*}} r(p(y))+\sum_{y=y^{*}+1}^{\bar{y}} r(p(y))=R(a, p, \bar{y})$, hence $R\left(a^{\prime}, p^{\prime}, \bar{y}\right)<R(a, p, \bar{y})$ 


\subsection{Proof of Theorem 2}

Let $(I, E)$ satisfy Assumption 1 and let $y^{s}$ be the serial production level in this economy. If $(a, p)$ is a standard pricing scheme, we use the notation

$$
R(a, p)=a+\sum_{y=1}^{\infty} r(p(y))
$$

to denote the revenue that it generates. If $(\mathbf{x}, \mathbf{y})$ is generated by $(a, p)$, we also have, using notation (9.9), $R(a, p)=R(a, p, \bar{y})$.

Part 1. Suppose $(\mathbf{x}, \mathbf{y})$ is efficient. We prove that conditions (1), (2) hold and that conditions (3), (4) hold if $\bar{y}>0$.

(1) By feasibility, $R(a, p) \geq C(\bar{y})$. Suppose, contrary to the claim, that this inequality is strict. Then, for any small enough $\varepsilon>0, R(a-\varepsilon, p) \geq C(\bar{y})$. The allocation $\left(\mathbf{x}^{\varepsilon}, \mathbf{y}^{\varepsilon}\right)$ given by $\mathbf{x}^{\varepsilon}(v)=\mathbf{x}(v)-\varepsilon, \mathbf{y}^{\varepsilon}(v)=\mathbf{y}(v)$ for all $v \in I$ Paretodominates $(\mathbf{x}, \mathbf{y})$ and is feasible since $\int_{I} \mathbf{x}^{\varepsilon} d E=R(a-\varepsilon, p) \geq C(\bar{y})=C\left(\mathbf{y}^{\varepsilon}(\bar{v})\right)$.

(2) Suppose, by contradiction, $\bar{y}<y^{s}$. By definition,

$$
p^{s}(\bar{y}+1)<p^{s}\left(y^{s}+1\right)=\bar{v}=p(\bar{y}+1) .
$$

Define $p^{\prime}: \mathbb{N} \backslash\{0\} \rightarrow I$ by $p^{\prime}(y)=p(y)$ for $y \leq \bar{y}$ and $p^{\prime}(y)=p^{s}(y)$ for $y \geq \bar{y}+1$. This mapping may fail to be nondecreasing as $p(\bar{y})$ may exceed $p^{s}(\bar{y}+1)$. However, let $\pi$ be a bijection from $\left\{1, \ldots, y^{s}\right\}$ onto itself such that the map $p^{\prime \prime}:\left\{1, \ldots, y^{s}\right\} \rightarrow I$ defined by $p^{\prime \prime}(y)=p^{\prime}(\pi(y))$ is non-decreasing; extend $p^{\prime \prime}$ to $\mathbb{N} \backslash\{0\}$ by setting $p^{\prime \prime}(y)=\bar{v}$ for $y \geq y^{s}+1$. By construction, $\left(a, p^{\prime \prime}\right)$ is a standard pricing scheme such that $\left(a, p^{\prime \prime}\right) \prec(a, p)$. Let $\left(\mathbf{x}^{\prime \prime}, \mathbf{y}^{\prime \prime}\right)$ be an allocation generated by $\left(a, p^{\prime \prime}\right)$ and 
such that $\bar{y}^{\prime \prime}=y^{s}$. By Lemma $2,\left(\mathbf{x}^{\prime \prime}, \mathbf{y}^{\prime \prime}\right)$ Pareto-dominates $(\mathbf{x}, \mathbf{y})$. Next,

$$
\begin{aligned}
R\left(a, p^{\prime \prime}\right) & =a+\sum_{y=1}^{y^{s}} r\left(p^{\prime \prime}(y)\right) \\
& =a+\sum_{y=1}^{y^{s}} r\left(p^{\prime}(\pi(y))\right) \\
& =a+\sum_{y=1}^{y^{s}} r\left(p^{\prime}(y)\right)(\text { since } \pi \text { is a bijection) } \\
& =a+\sum_{y=1}^{\bar{y}} r(p(y))+\sum_{y=\bar{y}+1}^{y^{s}} r\left(p^{s}(y)\right) \\
& =R(a, p)+\sum_{y=\bar{y}+1}^{y^{s}} r\left(p^{s}(y)\right) \\
& =C(\bar{y})+\sum_{y=\bar{y}+1}^{y^{s}} r\left(p^{s}(y)\right) \text { (since (x, y) is feasible) } \\
& =C(\bar{y})+\sum_{y=\bar{y}+1}^{y^{s}} c(y)(\text { by definition of serial allocations) } \\
& =C\left(y^{s}\right) .
\end{aligned}
$$

Thus, $\left(\mathbf{x}^{\prime \prime}, \mathbf{y}^{\prime \prime}\right)$ is feasible, contradicting the assumption that $(\mathbf{x}, \mathbf{y})$ is efficient.

(3) Assume $\bar{y}>0$. Suppose, by contradiction, that $p(\bar{y})>v^{*}$. Define $p^{\prime}(y)=$ $\min \left\{p(y), v^{*}\right\}$ if $y \leq \bar{y}, p^{\prime}(y)=p(y)$ if $y \geq \bar{y}+1$. Then $\left(a, p^{\prime}\right)$ is a standard pricing scheme, $\left(a, p^{\prime}\right) \prec(a, p)$, and $R\left(a, p^{\prime}\right)=a+\sum_{y=1}^{\bar{y}} r\left(\min \left\{p(y), v^{*}\right\}\right)>$ $a+\sum_{y=1}^{\bar{y}} r(p(y))=R(a, p)$. Choose an allocation $\left(\mathbf{x}^{\prime}, \mathbf{y}^{\prime}\right)$ generated by $\left(a, p^{\prime}\right)$ such that $\mathbf{y}^{\prime}(\bar{v})=\bar{y}$. Since $\left(a, p^{\prime}\right) \prec(a, p)$, Lemma 1 implies that $\left(\mathbf{x}^{\prime}, \mathbf{y}^{\prime}\right)$ Paretodominates $(\mathbf{x}, \mathbf{y})$. But $\left(\mathbf{x}^{\prime}, \mathbf{y}^{\prime}\right)$ is feasible since $\int_{I} \mathbf{x}^{\prime} d E=R\left(a, p^{\prime}\right) \geq R(a, p)=$ $C(\bar{y})=C\left(\mathbf{y}^{\prime}(\bar{v})\right)$.

(4) Assume $\bar{y}>0$. Let $\left(a^{1}, p^{1}\right)$ be the standard pricing scheme defined just before Theorem 2. Contrary to the claim, suppose $a^{1}+\sum_{y=1}^{\bar{y}-1} r\left(p^{1}(y)\right) \geq C(\bar{y}-1)$ : the allocation $\left(\mathbf{x}^{1}, \mathbf{y}^{1}\right)$ represented by $\left(a^{1}, p^{1}\right)$ and such that $\bar{y}^{1}=\bar{y}-1$ is then feasible. By condition 3), $p(\bar{y}) \leq v^{*}<\bar{v}$. Then $\left(a^{1}, p^{1}\right) \prec(a, p)$ and, by Lemma 1 , $\left(\mathbf{x}^{1}, \mathbf{y}^{1}\right)$ Pareto-dominates $(\mathbf{x}, \mathbf{y})$, again a contradiction. 
Part 2. Suppose that conditions (1), (2) hold and that conditions (3), (4) hold if $\bar{y}>0$. We show that $(\mathbf{x}, \mathbf{y})$ is efficient.

The proof is divided into four steps.

Step 1. No $\left(\mathbf{x}^{\prime}, \mathbf{y}^{\prime}\right) \in A^{C}(I, E)$ such that $\bar{y}^{\prime} \geq \bar{y}$ Pareto-dominates $(\mathbf{x}, \mathbf{y})$.

Suppose $\left(\mathbf{x}^{\prime}, \mathbf{y}^{\prime}\right) \in A(I, E)$ Pareto-dominates $(\mathbf{x}, \mathbf{y})$. Let $(a, p),\left(a^{\prime}, p^{\prime}\right)$ be the standard pricing schemes representing $(\mathbf{x}, \mathbf{y}),\left(\mathbf{x}^{\prime}, \mathbf{y}^{\prime}\right)$. By Lemma $1,\left(a^{\prime}, p^{\prime}\right) \prec$ $(a, p)$.

If $\bar{y}^{\prime}=\bar{y}$, Lemma 2 directly implies $R\left(a^{\prime}, p^{\prime}\right)<R(a, p)$, hence $\left(\mathbf{x}^{\prime}, \mathbf{y}^{\prime}\right)$ is not feasible.

If $\bar{y}^{\prime}>\bar{y}$, Lemma 2 implies

$$
R\left(a^{\prime}, p^{\prime}, \bar{y}\right)<R(a, p, \bar{y})=C(\bar{y}) .
$$

Since $\bar{y} \geq y^{s}$, we have $r(v)<c(y)$ for all $v \in I$ and all $y \geq \bar{y}+1$, hence, $\sum_{y=\bar{y}+1}^{\bar{y}^{\prime}} r\left(p^{\prime}(y)\right)<\sum_{y=\bar{y}+1}^{\bar{y}^{\prime}} c(y)$. Given (9.12),

$$
\begin{aligned}
R\left(a^{\prime}, p^{\prime}\right) & =R\left(a^{\prime}, p^{\prime}, \bar{y}\right)+\sum_{y=\bar{y}+1}^{\bar{y}^{\prime}} r\left(p^{\prime}(y)\right) \\
& <C(\bar{y})+\sum_{y=\bar{y}+1}^{\bar{y}^{\prime}} c(y) \\
& =C\left(\bar{y}^{\prime}\right),
\end{aligned}
$$

that is, $\left(\mathbf{x}^{\prime}, \mathbf{y}^{\prime}\right)$ is not feasible.

Step 2. No $\left(\mathbf{x}^{\prime}, \mathbf{y}^{\prime}\right) \in A^{C}(I, E)$ such that $\bar{y}^{\prime}=\bar{y}-1$ Pareto-dominates $(\mathbf{x}, \mathbf{y})$.

Suppose $\left(\mathbf{x}^{\prime}, \mathbf{y}^{\prime}\right) \in A(I, E)$ Pareto-dominates $(\mathbf{x}, \mathbf{y})$ and $\bar{y}^{\prime}=\bar{y}-1$. Let $(a, p)$, $\left(a^{\prime}, p^{\prime}\right)$ be the standard pricing schemes representing $(\mathbf{x}, \mathbf{y}),\left(\mathbf{x}^{\prime}, \mathbf{y}^{\prime}\right)$. By Lemma $1,\left(a^{\prime}, p^{\prime}\right) \prec(a, p)$. Next, since $\mathbf{y}(\bar{v})=\bar{y}$ and $\mathbf{y}^{\prime}(\bar{v})=\bar{y}^{\prime}$, the requirement that $\bar{v} \mathbf{y}^{\prime}(\bar{v})-\mathbf{x}^{\prime}(\bar{v}) \geq \bar{v} \mathbf{y}(\bar{v})-\mathbf{x}(\bar{v})$ reads

$$
a^{\prime}+\sum_{y=1}^{\bar{y}-1} p^{\prime}(y) \leq a+\sum_{y=1}^{\bar{y}} p(y)-\bar{v} .
$$

By definition of $\left(a^{1}, p^{1}\right)$, we have $\left(a^{\prime}, p^{\prime}\right) \preceq\left(a^{1}, p^{1}\right)$. By Lemma $2, R\left(a^{\prime}, p^{\prime}\right) \leq$ $R\left(a^{1}, p^{1}\right)$. By condition (4), $R\left(a^{1}, p^{1}\right)<C(\bar{y}-1)$, hence $R\left(a^{\prime}, p^{\prime}\right)<C(\bar{y}-1)=$ $C\left(\bar{y}^{\prime}\right)$, that is, $\left(\mathbf{x}^{\prime}, \mathbf{y}^{\prime}\right)$ is not feasible. 
Step 3. No $\left(\mathbf{x}^{\prime}, \mathbf{y}^{\prime}\right) \in A^{C}(I, E)$ such that $\bar{y}^{\prime}<\bar{y}$ Pareto-dominates $(\mathbf{x}, \mathbf{y})$.

The argument is by induction: if no $\left(\mathbf{x}^{\prime}, \mathbf{y}^{\prime}\right) \in A^{C}(I, E)$ such that $\bar{y}^{\prime}=k$ Pareto-dominates $(\mathbf{x}, \mathbf{y})$, then no $\left(\mathbf{x}^{\prime}, \mathbf{y}^{\prime}\right) \in A^{C}(I, E)$ such that $\bar{y}^{\prime}=k-1$ Paretodominates $(\mathbf{x}, \mathbf{y})$. For definiteness, we prove the claim for $k=\bar{y}-1$.

Denote by $\left(a^{2}, p^{2}\right)$ the $(\preceq-)$ maximal standard pricing scheme $\left(a^{\prime \prime}, p^{\prime \prime}\right)$ such that $\left(a^{\prime \prime}, p^{\prime \prime}\right) \preceq(a, p)$ and

$$
a^{\prime \prime}+\sum_{y=1}^{\bar{y}-2} p^{\prime \prime}(y) \leq a+\sum_{y=1}^{\bar{y}} p(y)-2 \bar{v} .
$$

Now, let $\left(\mathbf{x}^{\prime \prime}, \mathbf{y}^{\prime \prime}\right) \in A(I, E)$ be an allocation Pareto-dominating $(\mathbf{x}, \mathbf{y})$ and such that $\bar{y}^{\prime \prime}=\bar{y}-2$; denote by $\left(a^{\prime \prime}, p^{\prime \prime}\right)$ its standard pricing scheme. We must show that $R\left(a^{\prime \prime}, p^{\prime \prime}\right)<C(\bar{y}-2)$.

By construction,

$$
R\left(a^{\prime \prime}, p^{\prime \prime}\right) \leq R\left(a^{2}, p^{2}\right) .
$$

Indeed, since $\left(\mathbf{x}^{\prime \prime}, \mathbf{y}^{\prime \prime}\right)$ Pareto-dominates $(\mathbf{x}, \mathbf{y})$, Lemma 1 yields $\left(a^{\prime \prime}, p^{\prime \prime}\right) \prec(a, p)$. Since $\mathbf{y}(\bar{v})=\bar{y}$ and $\mathbf{y}^{\prime \prime}(\bar{v})=\bar{y}^{\prime \prime}$, the requirement that $\bar{v} \mathbf{y}^{\prime \prime}(\bar{v})-\mathbf{x}^{\prime \prime}(\bar{v}) \geq \bar{v} \mathbf{y}(\bar{v})-\mathbf{x}(\bar{v})$ reads $a^{\prime \prime}+\sum_{y=1}^{\bar{y}-2} p^{\prime \prime}(y) \leq a+\sum_{y=1}^{\bar{y}} p(y)-2 \bar{v}$. Thus $\left(a^{\prime \prime}, p^{\prime \prime}\right) \preceq\left(a^{2}, p^{2}\right)$ by definition of $\left(a^{2}, p^{2}\right)$, and (9.13) follows by Lemma 2. Given (9.13), it suffices to prove that

$$
R\left(a^{2}, p^{2}\right)<C(\bar{y}-2) .
$$

The proof of (9.14) relies on the following claim:

$$
R(a, p)-R\left(a^{1}, p^{1}\right) \leq R\left(a^{1}, p^{1}\right)-R\left(a^{2}, p^{2}\right)
$$

or equivalently

$$
R(a, p)+R\left(a^{2}, p^{2}\right) \leq 2 R\left(a^{1}, p^{1}\right) .
$$

By construction of $\left(a^{1}, p^{1}\right)$ there exists a consumption level $k \in\{1, \ldots, \bar{y}-1\}$ such that $p^{1}(y)=p(y)$ if $1 \leq y<k$ and $p^{1}(y)=\lambda^{1}$ if $k \leq y \leq \bar{y}-1$. By construction of $\left(a^{2}, p^{2}\right)$, there exists $l \in\{1, \ldots, \bar{y}-2\}$ such that $p^{2}(y)=p(y)$ if $1 \leq y<l$ and $p^{2}(y)=\lambda^{2}$ if $l \leq y \leq \bar{y}-2$. Clearly, $\ell \leq k$. If $\ell=k$, then, by convention, $\{\ell, \ldots, k-1\}=\emptyset$. Only three cases can occur.

Case 1. $a=a^{1}=a^{2}$. Then claim (9.15) reads 
$\left(a+\sum_{y=1}^{\bar{y}} r(p(y))\right)+\left(a+\sum_{y=1}^{\ell-1} r(p(y))+\sum_{y=\ell}^{\bar{y}-2} r\left(\lambda^{2}\right)\right) \leq 2\left(a+\sum_{y=1}^{k-1} r(p(y))+\sum_{y=k}^{\bar{y}-1} r\left(\lambda^{1}\right)\right)$.

To prove (9.16), note first that $\sum_{y=k}^{\bar{y}-1}\left(p(y)-\lambda^{1}\right)=\bar{v}-p(\bar{y})$ and $\sum_{y=l}^{\bar{y}-2}\left(p^{1}(y)-\right.$ $\left.\lambda^{2}\right)=\bar{v}-p^{1}(\bar{y}-1)$. Combining these two equations, we get

$$
\begin{aligned}
\sum_{y=l}^{\bar{y}-2} \lambda^{2} & =\sum_{y=l}^{\bar{y}-1} p^{1}(y)+\sum_{y=k}^{\bar{y}-1} \lambda^{1}-\sum_{y=k}^{\bar{y}} p(y) \\
& =\sum_{y=l}^{k-1} p(y)+2 \sum_{y=k}^{\bar{y}-1} \lambda^{1}-\sum_{y=k}^{\bar{y}} p(y)
\end{aligned}
$$

hence,

$$
\sum_{y=l}^{\bar{y}} p(y)+\sum_{y=l}^{\bar{y}-2} \lambda^{2}=2\left(\sum_{y=l}^{k-1} p(y)+\sum_{y=k}^{\bar{y}-1} \lambda^{1}\right) .
$$

There are $2(\bar{y}-\ell)$ terms on each side of this equation. Listing them in nondecreasing order, we get

$$
q=(\underbrace{\lambda^{2}, \ldots, \lambda^{2}}_{\bar{y}-\ell-1}, \underbrace{p(l), \ldots, p(k-1)}_{k-l} \underbrace{p(k), \ldots, p(\bar{y})}_{\bar{y}-k+1})
$$

on the left-hand side and

$$
q^{\prime}=(\underbrace{p(\ell), p(\ell), \ldots, p(k-1), p(k-1)}_{2(k-l)} \underbrace{\lambda^{1}, \ldots, \lambda^{1}}_{2(\bar{y}-k)})
$$

on the right-hand side. Observe that $q_{j} \leq q_{j}^{\prime}$ for all $j \leq \bar{y}-2 \ell+k-1$ and $q_{j} \geq q_{j}^{\prime}$ for all $j>\bar{y}-2 \ell+k-1$. Therefore, $\sum_{j=1}^{2(\bar{y}-\ell)} q_{j}=\sum_{j=1}^{2(\bar{y}-\ell)} q_{j}^{\prime}$ and $\sum_{j=1}^{k} q_{j} \leq \sum_{i=1}^{k} q_{j}^{\prime}$ for all $k \in\{1, \ldots, 2(\bar{y}-\ell)\}$. By the Hardy, Littlewood and Polya theorem, $\sum_{j=1}^{2(\bar{y}-\ell)} r\left(q_{j}\right) \leq \sum_{j=1}^{2(\bar{y}-\ell)} r\left(q_{j}^{\prime}\right)$, that is,

$$
\sum_{y=\ell}^{\bar{y}} r(p(y))+\sum_{y=\ell}^{\bar{y}-2} r\left(\lambda^{2}\right) \leq 2\left(\sum_{y=\ell}^{k-1} r(p(y))+\sum_{y=k}^{\bar{y}-1} r\left(\lambda^{1}\right)\right) .
$$


Adding $2\left(a+\sum_{y=1}^{l-1} r(p(y))\right.$ on both sides yields (9.16).

Case 2. $a=a^{1}>a^{2}$. Then $p^{2}(y)=\underline{v}$ for $1 \leq y \leq \bar{y}-2$ and claim (9.15) reads

$$
\sum_{y=1}^{\bar{y}} r(p(y))+\sum_{y=\ell}^{\bar{y}-2} r(\underline{v}) \leq\left(a-a^{2}\right)+2\left(\sum_{y=1}^{k-1} r(p(y))+\sum_{y=k}^{\bar{y}-1} r\left(\lambda^{1}\right)\right) .
$$

The argument is essentially the same as in Case 1 . Note that $\sum_{y=k}^{\bar{y}-1}(p(y)-$ $\left.\lambda^{1}\right)=\bar{v}-p(\bar{y})$ and $\left(a+\sum_{y=1}^{k-1} p(y)+\sum_{y=k}^{\bar{y}-2} \lambda^{1}\right)-\left(a^{2}+\sum_{y=1}^{\bar{y}-2} \underline{v}\right)=\bar{v}-\lambda^{1}$. Combining these two equations, we get

$$
\sum_{y=1}^{\bar{y}} p(y)+\sum_{y=1}^{\bar{y}-2} \underline{v}=\left(a-a^{2}\right)+2\left(\sum_{y=1}^{k-1} p(y)+\sum_{y=k}^{\bar{y}-1} \lambda^{1}\right),
$$

hence,

$$
\sum_{y=1}^{\bar{y}} p(y)+\sum_{y=1}^{\bar{y}-2} \underline{v} \leq 2\left(\sum_{y=1}^{k-1} p(y)+\sum_{y=k}^{\bar{y}-1} \lambda^{1}\right) .
$$

Listing the There are $2 \bar{y}-2$ terms on each side of this inequality in nondecreasing order, we get

$$
q=(\underbrace{\underline{v}, \ldots, \underline{v}}_{\bar{y}-2}, \underbrace{p(1), \ldots, p(\bar{y})}_{\bar{y}})
$$

on the left-hand side and

$$
q^{\prime}=(\underbrace{p(1), p(1), \ldots, p(k-1), p(k-1)}_{2(k-1)} \underbrace{\lambda^{1}, \ldots, \lambda^{1}}_{2(\bar{y}-k)})
$$

on the right-hand side. Since $q_{j} \leq q_{j}^{\prime}$ for all $j \leq \bar{y}+k-3$ and $q_{j} \geq q_{j}^{\prime}$ for all $j>\bar{y}+k-3$, we have $\sum_{j=1}^{k} q_{j} \leq \sum_{i=1}^{k} q_{j}^{\prime}$ for all $k \in\{1, \ldots, 2 \bar{y}-2\}$ and the Hardy, Littlewood and Polya theorem yields $\sum_{j=1}^{2 \bar{y}-2} r\left(q_{j}\right) \leq \sum_{j=1}^{2 \bar{y}-2} r\left(q_{j}^{\prime}\right)$, that is,

$$
\sum_{y=1}^{\bar{y}} r(p(y))+\sum_{y=\ell}^{\bar{y}-2} r(\underline{v}) \leq 2\left(\sum_{y=1}^{k-1} r(p(y))+\sum_{y=k}^{\bar{y}-1} r\left(\lambda^{1}\right)\right)
$$


hence, a fortiori, (9.17).

Case 3. $a>a^{1}>a^{2}$. Then $p^{1}(y)=\underline{v}$ for $1 \leq y \leq \bar{y}-1$ and $p^{2}(y)=\underline{v}$ for $1 \leq$ $y \leq \bar{y}-2$. Under the scheme $\left(a^{1}, p^{1}\right)$, almost all agents consume $\bar{y}-1$ and pay $a^{1}+$ $\underline{v}(\bar{y}-1)=a+\sum_{y=1}^{\bar{y}} p(y)-\bar{v}$, hence $R\left(a^{1}, p^{1}\right)=a+\sum_{y=1}^{\bar{y}} p(y)-\bar{v}$. Likewise, under $\left(a^{2}, p^{2}\right)$ almost all agents consume $\bar{y}-2$ and pay $a^{2}+\underline{v}(\bar{y}-2)=a+\sum_{y=1}^{\bar{y}} p(y)-2 \bar{v}$, hence $R\left(a^{2}, p^{2}\right)=a+\sum_{y=1}^{\bar{y}} p(y)-2 \bar{v}$. Therefore, $R\left(a^{1}, p^{1}\right)-R\left(a^{2}, p^{2}\right)=\bar{v}$. Next, $R(a, p)=a+\sum_{y=1}^{\bar{y}} r(p(y)) \leq a+\sum_{y=1}^{\bar{y}} p(y)$, hence $R(a, p)-R\left(a^{1}, p^{1}\right) \leq \bar{v}$.

We have completed the proof of (9.15). Recalling from Step 2 that $R\left(a^{1}, p^{1}\right)<$ $C(\bar{y}-1)$ and since $R(a, p)=C(\bar{y}),(9.15)$ implies $R\left(a^{2}, p^{2}\right) \leq 2 R\left(a^{1}, p^{1}\right)-R(a, p)$ $<2 C(\bar{y}-1)-C(\bar{y}) \leq C(\bar{y}-2)$. This proves (9.14) and the theorem.

\subsection{Proof of Theorem 3}

Let $\varphi$ be an allocation rule on $\mathcal{L}^{1}$.

Part 1. Suppose $\varphi$ is a serial rule. We show that $\varphi$ satisfies efficiency and demand monotonicity.

(a) To check efficiency, let $(I, E) \in \mathcal{L}^{1}$ and let $(\mathbf{x}, \mathbf{y})=\varphi(I, E)$ be represented by the standard serial pricing scheme $\left(a^{s}, p^{s}\right)$. Conditions 1 to 3 in Theorem 2 are obviously met. To check condition 4 , let $\left(a^{1}, p^{1}\right)$ be the maximal scheme $\left(a^{\prime}, p^{\prime}\right) \preceq\left(a^{s}, p^{s}\right)$ such that $a^{\prime}+\sum_{y=1}^{y^{s}-1} p^{\prime}(y) \leq a^{s}+\sum_{y=1}^{y^{s}} p^{s}(y)-\bar{v}$. By construction, there exists $k \in\{1, \ldots, \bar{y}-1\}$ such that $p^{1}(y)=p^{s}(y)$ if $1 \leq y<k$ and $p^{1}(y)=$ $\lambda^{1}$ if $k \leq y \leq \bar{y}-1$, where $\lambda^{1}<p^{s}(k)$. By Lemma 2 , $a^{1}+\sum_{y=1}^{y^{s}-1} r\left(p^{1}(y)\right)=$ $a^{1}+\sum_{y=1}^{k-1} r\left(p^{s}(y)\right)+\sum_{y k}^{y^{s}-1} r\left(\lambda^{1}\right)<a^{s}+\sum_{y=1}^{y^{s}-1} r\left(p^{s}(y)\right)=C\left(y^{s}-1\right)$.

(b) To check demand monotonicity, let $\left(I^{1}, E^{1}\right),\left(I^{2}, E^{2}\right) \in \mathcal{L}^{1}$ be such that $\left(I^{1}, E^{1}\right) \unlhd\left(I^{2}, E^{2}\right)$. For $i=1,2$, let $\left(\mathbf{x}^{i}, \mathbf{y}^{i}\right)=\varphi\left(I^{i}, E^{i}\right)$ be represented by the standard serial pricing scheme $\left(a^{s}\left(E^{i}\right), p^{s}\left(., E^{i}\right)\right)$ and, for every $y \in \mathbb{N} \backslash\{0\}$, denote the smallest valuation $v \in \mathbb{R}_{+}$such that $r\left(v, E^{i}\right) \geq c(y)$ by $v^{s}\left(y, E^{i}\right)$. Since $\left(I^{1}, E^{1}\right) \unlhd\left(I^{2}, E^{2}\right)$, we have $r\left(v, E^{1}\right) \leq r\left(v, E^{2}\right)$ for all $v \in \mathbb{R}_{+}$, hence $v^{s}\left(y, E^{1}\right) \geq$ $v^{s}\left(y, E^{1}\right)$ for all $y \in \mathbb{N} \backslash\{0\}$. It follows that $\left(a^{s}\left(E^{2}\right), p^{s}\left(., E^{2}\right)\right) \preceq\left(a^{s}\left(E^{1}\right), p^{s}\left(., E^{1}\right)\right)$ and therefore $v \mathbf{y}^{2}(v)-\mathbf{x}^{2}(v) \geq v \mathbf{y}^{1}(v)-\mathbf{x}^{1}(v)$ for all $v \in I^{1} \cap I^{2}$.

Part 2. Suppose $\varphi$ satisfies efficiency and demand monotonicity. We show that it is serial.

For every $k \in \mathbb{N} \backslash\{0\}$, define

$$
\mathcal{L}^{1}(k)=\left\{(I, E) \in \mathcal{L}^{1} \mid \max _{v \in I} r(v, E) \leq c(k)\right\} .
$$


The proof is by induction on $k$.

Step 1. If $(I, E) \in \mathcal{L}^{1}(1)$, then $\varphi(I, E)$ is a serial allocation serial for $(I, E)$.

Let $(I, E) \in \mathcal{L}^{1}$, let $(\mathbf{x}, \mathbf{y})=\varphi(I, E)$, and let $(a, p)$ be the standard pricing scheme representing $(\mathbf{x}, \mathbf{y})$. Let $\left(a^{s}, p^{s}\right)$ be the standard serial pricing scheme in the economy $(I, E)$. Since $\max _{v \in I} r(v, E) \leq c(1)$, we have $\underline{v} \leq c(1)$, hence, $a^{s}=0$, $p^{s}(1)=v^{s}(1), p^{s}(y)=\bar{v}$ for $y \geq 2$.

(a) We show that

$$
\bar{v}<c(1) \Rightarrow(\mathbf{x}(v), \mathbf{y}(v))=(0,0) \text { for all } v \in I .
$$

Suppose $\bar{v}<c(1)$. If $\bar{y}>0$, then $a+\sum_{y=1}^{\bar{y}} r(p(y), E) \geq C(\bar{y})$ implies $a+$ $\sum_{y=1}^{\bar{y}} p(y) \geq C(\bar{y})$, hence $\bar{v} \mathbf{y}(\bar{v})-\mathbf{x}(\bar{v})=\overline{v y}-\left(a+\sum_{y=1}^{\bar{y}} p(y)\right) \leq \overline{v y}-C(\bar{y})<0$ since $\bar{v}<c(1) \leq c(y)$ for all $y>1$. Then, for all $v \in I, v \mathbf{y}(v)-\mathbf{x}(v) \leq \bar{v} \mathbf{y}(v)-\mathbf{x}(v)$ $\leq \bar{v} \mathbf{y}(\bar{v})-\mathbf{x}(\bar{v})<0$. Thus, the allocation $\left(\mathbf{x}^{\prime}, \mathbf{y}^{\prime}\right)$ given by $\left(\mathbf{x}^{\prime}(v), \mathbf{y}^{\prime}(v)\right)=(0,0)$ for all $v \in I$ Pareto-dominates $(\mathbf{x}, \mathbf{y})$, a contradiction. Hence $\bar{y}=0$. Since $(\mathbf{x}, \mathbf{y})$ is incentive-compatible, $(\mathbf{x}(v), \mathbf{y}(v))=(0,0)$ for all $v \in I$.

(b) We show that

$$
\max _{v \in I} r(v, E)<c(1) \Rightarrow(\mathbf{x}(v), \mathbf{y}(v))=(0,0) \text { for all } v \in I .
$$

Suppose $\max _{v \in I} r(v, E)<c(1)$. Then $\underline{v}<c(1)$. We can therefore construct an economy $\left(I^{1}, E^{1}\right) \in \mathcal{E}^{1}(1)$ such that $\left(I^{1}, E^{1}\right) \unlhd(I, E), \underline{v} \in I^{1}$, and $v<c(1)$ for all $v \in I^{1}$. For instance, let

$$
E^{1}(v)=\min \{\alpha E(v), 1\} \text { for all } v \in \mathbb{R}_{+},
$$

where $\alpha>1$ is large enough. Note that $\left(I^{1}, E^{1}\right)$ satisfies Assumption L1 because $(I, E)$ does. Let $\varphi\left(I^{1}, E^{1}\right)=\left(\mathbf{x}^{1}, \mathbf{y}^{1}\right)$. By $(9.18),\left(\mathbf{x}^{1}(v), \mathbf{y}^{1}(v)\right)=(0,0)$ for all $v \in I^{1}$. Hence, by demand monotonicity,

$$
v \mathbf{y}(v)-\mathbf{x}(v) \geq 0 \text { for all } v \in I \cap I^{1} .
$$

Suppose $\bar{y}>0$. Since $r(p(y), E)<c(y)$ for all $y=1, \ldots, \bar{y}$, feasibility requires $a>0$. Since $p(y) \geq \underline{v}$ for all $y=1, \ldots, \bar{y}$, we have $\underline{v} \mathbf{y}(\underline{v})-\mathbf{x}(\underline{v})=-a<0$, contradicting (9.21) since $\underline{v} \in I \cap I^{1}$. Thus, $\bar{y}=0$, hence, $(\mathbf{x}(v), \mathbf{y}(v))=(0,0)$ for all $v \in I$.

(c) We show that $(\mathbf{x}, \mathbf{y})$ is a serial allocation for $(I, E)$. From (b), we know that this is true if $\max _{v \in I} r(v, E)<c(1)$. Assume now that $\max _{v \in I} r(v, E)=c(1)$. In this case, the serial production level is $y^{s}=1$. By Theorem $2, \bar{y} \geq 1$. 
Construct an economy $\left(I^{2}, E^{2}\right) \in \mathcal{L}^{1}(1)$ such that $\left(I^{2}, E^{2}\right) \unlhd(I, E), \underline{v} \in I^{2}$, and $\max _{v \in I^{2}} r\left(v, E^{2}\right)<c(1)$. Such an economy always exists, even though an explicit construction requires to distinguish two cases. ${ }^{9}$ If $r(\underline{v}, E)<c(1)$, simply let $E^{2}=E^{1}$, as defined in (9.20). If $r(\underline{v}, E)=c(1)$, note that $\underline{v}>0$. Choose $\varepsilon$, $0<\varepsilon<\underline{v}$, and let

$$
E^{2}(v)=E(v+\varepsilon) \text { for all } v \in \mathbb{R}_{+} .
$$

Let $\varphi\left(I^{2}, E^{2}\right)=\left(\mathbf{x}^{2}, \mathbf{y}^{2}\right)$. By $(9.19),\left(\mathbf{x}^{2}(v), \mathbf{y}^{2}(v)\right)=(0,0)$ for all $v \in I^{2}$. Hence, by demand monotonicity,

$$
v \mathbf{y}(v)-\mathbf{x}(v) \geq 0 \text { for all } v \in I \cap I^{2} .
$$

Suppose $\bar{y}>1$. Since $r(p(1), E) \leq c(1)$ and $r(p(y), E)<c(y)$ for all $y=2, \ldots, \bar{y}$, feasibility requires $a>0$, hence again,

$$
\underline{v} \mathbf{y}(\underline{v})-\mathbf{x}(\underline{v})=-a<0,
$$

contradicting (9.22) since $\underline{v} \in I \cap I^{2}$.

Thus $\bar{y}=1$. Since $r(p(1), E) \leq c(1)$, feasibility requires $a \geq 0$. Since $a>0$ implies (9.23), which is a contradiction, we must have $a=0$. Then feasibility forces $r(p(1), E)=c(1)$ and since $p(1) \leq v^{*}$ by Theorem 2, $p(1)$ is the smallest $v \in I$ such that $r(v, E) \geq c(1)$. This means that $(a, p)$ is the standard serial pricing scheme $\left(a^{s}, p^{s}\right)$, and $(\mathbf{x}, \mathbf{y})$ is serial.

Step 2. For every $k \in \mathbb{N} \backslash\{0\}$, if $\varphi(I, E)$ is a serial allocation for all $(I, E) \in \mathcal{E}^{1}(k)$, then $\varphi(I, E)$ is a serial allocation for all $(I, E) \in \mathcal{L}^{1}(k+1)$.

Fix $k \in \mathbb{N} \backslash\{0\}$ and make the induction hypothesis that

$$
\text { for all }(I, E) \in \mathcal{L}^{1}(k), \varphi(I, E) \text { is a serial allocation for }(I, E) \text {. }
$$

Fix $(I, E) \in \mathcal{L}^{1}(k+1) \backslash \mathcal{L}(k)$. That is, $c(k)<\max _{v \in I} r(v, E) \leq c(k+1)$. Let $\varphi(I, E)=(\mathbf{x}, \mathbf{y})$, let $(a, p)$ be the standard pricing scheme representing $(\mathbf{x}, \mathbf{y})$, and let $\left(a^{s}, p^{s}\right)$ be the standard serial pricing scheme in the economy $(I, E)$.

For $y=1, \ldots, k$, denote by $v^{s}(y)$ the smallest valuation $v \in \mathbb{R}_{+}$such that $r(v, E) \geq c(y)$. This is well defined because $c(k)<\max _{v \in I} r(v, E)$. Define

$$
\begin{aligned}
E^{1}(v) & =E(v) \text { if } v \leq v^{s}(k), \\
& =\min \left\{E\left(v^{s}(k)\right)+\frac{1}{\varepsilon}\left(v-v^{s}(k)\right),(1-\varepsilon)+\frac{\varepsilon}{\bar{v}} v, 1\right\} \text { if } v>v^{s}(k),
\end{aligned}
$$

\footnotetext{
${ }^{9}$ Let $I^{2}=\left[\underline{v}^{2}, \bar{v}^{2}\right]$. If $r(\underline{v}, E)<c(1)$ and $\underline{v}=0$, we must have $\underline{v}^{2}=0$. But if $r(\underline{v}, E)=c(1)$, the requirement that $\max _{v \in I^{2}} r\left(v, E^{2}\right)<c\left(\overline{1)}\right.$ forces $\underline{v}^{2}<\underline{v}$.
} 
where $\varepsilon>0$ is small enough to guarantee ${ }^{10}$ that $\left(I^{1}, E^{1}\right) \unlhd(I, E)$ and $\max _{v \in I^{1}} r\left(v, E^{1}\right)=$ $r\left(v^{s}(k), E\right)$. Let $I^{1}=\left[\underline{v}^{1}, \bar{v}^{1}\right]$ be the largest interval on which $E^{1}$ is strictly increasing. By construction, $\underline{v}^{1}=\min \left\{\underline{v}, v^{s}(k)\right\}$ and $\bar{v}^{1}=\bar{v}$, hence, $I^{1} \supseteq I$.

Observe also that $r\left(., E^{1}\right)$ coincides with $r(., E)$ on $\left[0, v^{s}(k)\right]$ since $E^{1}$ coincides with $E$ on that interval. Therefore the smallest valuation $v \in \mathbb{R}_{+}$such that $r\left(v, E^{1}\right) \geq c(y)$, denoted $v^{s}\left(y, E^{1}\right)$, coincides with $v^{s}(y)$ for $y=1, \ldots, k$. Hence the nondecreasing pricing scheme

$$
\begin{aligned}
a^{1} & =0, \\
p^{1}(y) & =v^{s}(y) \text { for all } 1 \leq y \leq k, \\
& =\overline{\bar{v}} \text { for } y \geq k+1,
\end{aligned}
$$

where $\overline{\bar{v}}>\bar{v}$, generates serial allocations for $\left(I^{1}, E^{1}\right)$-though not necessarily all of them. Moreover, $\left(I^{1}, E^{1}\right)$ satisfies Assumption L1 and $\left(I^{1}, E^{1}\right) \in \mathcal{L}^{1}(k)$.

Let $\varphi\left(I^{1}, E^{1}\right)=\left(\mathbf{x}^{1}, \mathbf{y}^{1}\right)$. By demand monotonicity,

$$
v \mathbf{y}(v)-\mathbf{x}(v) \geq v \mathbf{y}^{1}(v)-\mathbf{x}^{1}(v) \text { for all } y \in I
$$

since $I \cap I^{1}=I$. By the induction hypothesis, $\left(\mathrm{x}^{1}, \mathbf{y}^{1}\right)$ is a serial allocation for $\left(I^{1}, E^{1}\right)$. It is therefore Pareto-equivalent to an allocation generated by $\left(a^{1}, p^{1}\right)$. Clearly, (9.24) implies

$$
(a, p) \preceq\left(a^{1}, p^{1}\right) .
$$

By Lemma 2 and the definition of $\left(a^{1}, p^{1}\right), a+\sum_{q=1}^{y} r(p(q), E) \leq \sum_{q=1}^{y} r\left(v^{s}(q), E\right)$ for $y=1, \ldots, k$, and in particular,

$$
a+\sum_{y=1}^{k} r(p(y), E) \leq \sum_{y=1}^{k} r\left(v^{s}(y), E\right)=C(k) .
$$

Distinguish two cases.

(a) $\max r(v, E)<c(k+1)$. Then $k$ is the serial production level in $(I, E)$ and $\left(a^{1}, p^{1}\right)$ generates serial allocations for $(I, E)$. By Theorem $2, \bar{y} \geq k$.

If $\bar{y}>k$, it follows from (9.26) that $a+\sum_{y=1}^{\bar{y}} r(p(y), E)=a+\sum_{y=1}^{k} r(p(y), E)$ $+\sum_{y=k+1}^{\bar{y}} r(p(y), E) \leq C(k)+\sum_{y=k+1}^{\bar{y}} r(p(y), E)<C(k)+\sum_{y=k+1}^{\bar{y}} c(y)=C(\bar{y})$, a contradiction to the feasibility of $(\mathbf{x}, \mathbf{y})$.

\footnotetext{
${ }^{10}$ Because $c(k)<\max _{v \in I} r(v, E), r(., E)$ is increasing at $v^{s}(k, E)$. This guarantees that the right derivative of $E$ at that point is bounded.
} 
Thus $\bar{y}=k$. Because $(a, p)$ is standard and $\left(a^{1}, p^{1}\right)$ generates serial allocations for $(I, E)$, (9.25) implies that $(a, p) \preceq\left(a^{s}, p^{s}\right)$. By Lemma 2 and feasibility of $(\mathbf{x}, \mathbf{y}),(a, p)=\left(a^{s}, p^{s}\right)$. Thus $(\mathbf{x}, \mathbf{y})$ is a serial allocation for $(I, E)$.

(b) $\max r(v, E)=c(k+1)$. Then $k+1$ is the serial production level in $(I, E)$ and the nondecreasing pricing scheme

$$
\begin{aligned}
a^{2} & =0, \\
p^{2}(y) & =v^{s}(y) \text { for all } 1 \leq y \leq k+1, \\
& =\overline{\bar{v}} \text { for } y \geq k+2,
\end{aligned}
$$

which coincides with $\left(a^{1}, p^{1}\right)$ everywhere but on unit $k+1$, generates serial allocations for $(I, E)$. By Theorem $2, \bar{y} \geq k+1$.

If $\bar{y}>k+1$, recall that $r(p(k+1)) \leq c(k+1)$ and $r(p(y))<c(y)$ for $y \geq k+2$. Combining these inequalities with (9.26), we get again $a+\sum_{y=1}^{\bar{y}} r(p(y), E)<C(\bar{y})$, a contradiction to the feasibility of $(\mathbf{x}, \mathbf{y})$.

Thus $\bar{y}=k+1$. Note that $p^{2}(k+1)$ is the smallest revenue-maximizing price in $(I, E)$; hence, by condition 3 in Theorem $2, p(k+1) \leq p^{2}(k+1)$. Thus (9.25) implies $(a, p) \preceq\left(a^{2}, p^{2}\right)$. Because $(a, p)$ is standard and $\left(a^{2}, p^{2}\right)$ generates serial allocations for $(I, E),(a, p) \preceq\left(a^{2}, p^{2}\right)$ implies that $(a, p) \preceq\left(a^{s}, p^{s}\right)$. By Lemma 2 and feasibility of $(\mathbf{x}, \mathbf{y}),(a, p)=\left(a^{s}, p^{s}\right)$. Thus $(\mathbf{x}, \mathbf{y})$ is a serial allocation for $(I, E)$

\subsection{Proof of Theorem 4}

The proof that the serial rules satisfy efficiency follows from Theorem 3. The proof that they satisfy population monotonicity follows the argument given in the text and is, therefore, omitted here.

Let $\varphi$ be an allocation rule on $\overline{\mathcal{L}}$ and let $(I, E) \in \overline{\mathcal{L}}$ be an economy of size $m$. Let $\varphi(I, E)=(\mathbf{x}, \mathbf{y})$ with associated production level $\bar{y}$ and standard pricing scheme $(a, p)$. The proof is divided in a series of successive claims.

(a) $\bar{v}<\frac{c}{m} \Rightarrow(\mathbf{x}(v), \mathbf{y}(v))=(0,0)$ for all $v \in I$.

The proof parallels point (a) in the proof of Theorem 3.

(b) $\underline{v} \mathbf{y}(\underline{v})-\mathbf{x}(\underline{v}) \geq 0$.

Assume not. Let $\left(I^{1}, E^{1}\right) \in \overline{\mathcal{L}}$ be such that $I^{1}=I$ and $E^{1}=\varepsilon E$, where $\varepsilon$ satisfies $\bar{v}<\frac{c}{\varepsilon m}$. Let $\varphi\left(I^{1}, E^{1}\right)=\left(\mathbf{x}^{1}, \mathbf{y}^{1}\right)$. By (a), $\left(\mathbf{x}^{1}(\underline{v}), \mathbf{y}^{1}(\underline{v})\right)=(0,0)$. By population monotonicity, $\underline{v} \mathbf{y}(\underline{v})-\mathbf{x}(\underline{v}) \geq \underline{v} \mathbf{y}^{1}(\underline{v})-\mathbf{x}^{1}(\underline{v})=0$.

(c) If $\max _{v \in I} r(v, E)<c$, then $\bar{y}=0$ and $(a, p)=\left(a^{s}, p^{s}\right)$. 
Assume, instead, that $\bar{y}=1$. Note first that if $\max _{v \in I} r(v, E)<c$, then $\underline{v}<c$. If $\mathbf{y}(\underline{v})=0$, then by $(\mathrm{b}), \mathbf{x}(\underline{v})=a \leq 0$. Therefore, by budget balance, $r(p, E)=$ $c-a$, a contradiction. If $\mathbf{y}(\underline{v})=1$, then $\mathbf{y}(v)=1$ and $\mathbf{x}(v)=c$ for all $v \in I$. Therefore, $\underline{v} \mathbf{y}(\underline{v})-\mathbf{x}(\underline{v})=\underline{v}-c<0$, contradicting (b). This proves that $\bar{y}=0$, which implies $\mathbf{x}(v)=0$ for all $v \in I$, that is, $(a, p)=\left(a^{s}, p^{s}\right)$.

(d) If $\max _{v \in I} r(v, E) \geq c$, then $\bar{y}=1$ and $(a, p)=\left(a^{s}, p^{s}\right)$.

The fact that $\bar{y}=1$ follows from efficiency. To see that the pricing scheme must be serial, distinguishing two cases.

Case 1: $\mathbf{y}(\underline{v})=1$. Then, $\mathbf{y}(v)=1$ and $\mathbf{x}(v)=c$ for all $v \in I$. By $(\mathrm{b}), \underline{v} \geq c$, so that $(a, p)=\left(a^{s}, p^{s}\right)$.

Case 2: $\mathbf{y}(\underline{v})=0$. Then $p \geq \underline{v}$ and $\mathbf{x}(\underline{v})=a$. By (b), $a \leq 0$. If $a=0$, then $p$ is such that $r(p, E)=c$ and $(a, p)=\left(a^{s}, p^{s}\right)$. Assume $a<0$. We will derive a contradiction. Define $\left(I^{1}, E^{1}\right) \in \overline{\mathcal{L}}$ by $I^{1}=[p, \bar{v}]$ and $E^{1}(v)=E(v)-E(p)$ for all $v \in I^{1}$. By construction, $r\left(v, E^{1}\right)=r(v, E)$ for all $v \in I^{1}$, so that $r\left(p, E^{1}\right)=r(p, E)=-a+c$. Let $\varphi\left(I^{1}, E^{1}\right)=\left(\mathbf{x}^{1}, \mathbf{y}^{1}\right)$. By efficiency, $\bar{y}^{1}=1$. Define $\left(\mathbf{x}^{2}, \mathbf{y}^{2}\right) \in A\left(I^{1}, E^{1}\right)$ by $\mathbf{x}^{2}(v)=\mathbf{x}(v)=a+p$ and $\mathbf{y}^{2}(v)=\mathbf{y}(v)=$ 1 for all $v \in I^{1}$. Note that $\left(\mathbf{x}^{2}, \mathbf{y}^{2}\right)$ is feasible but not efficient (actually, not balanced) for $\left(I^{1}, E^{1}\right)$. Let $\varphi\left(I^{1}, E^{1}\right)=\left(\mathbf{x}^{3}, \mathbf{y}^{3}\right)$. By population monotonicity, $v \mathbf{y}^{3}(v)-\mathbf{x}^{3}(v) \leq v \mathbf{y}^{1}(v)-\mathbf{x}^{1}(v)=v \mathbf{y}^{2}(v)-\mathbf{x}^{2}(v)$ for all $v \in I^{1}$, contradicting efficiency.

\subsection{Proof of Theorem 6}

Let $\varphi$ be an allocation rule on $\mathcal{Q} \mathcal{L}^{1}$.

Part 1. Suppose $\varphi$ is a serial rule. The proof that it satisfies demand monotonicity is similar to the corresponding part of the proof of Theorem 3 and is therefore omitted. We show that $\varphi$ satisfies efficiency.

Rather than deriving this fact from a general characterization of efficient allocations as in the linear case, we provide a direct proof. We begin by characterizing Pareto dominance. Let $(T, E) \in \mathcal{Q} \mathcal{L}^{1}$ and let $(\mathbf{x}, \mathbf{y}),\left(\mathbf{x}^{\prime}, \mathbf{y}^{\prime}\right) \in A(T, E)$ be represented by their standard pricing schemes $(a, p),\left(a^{\prime}, p^{\prime}\right)$. As in the linear domain case, $\left(\mathbf{x}^{\prime}, \mathbf{y}^{\prime}\right)$ Pareto-dominates $(\mathbf{x}, \mathbf{y})$ if and only if $\left(a^{\prime}, p^{\prime}\right) \prec(a, p)$. The proof is similar to the proof of Lemma 1 , provided the set $K$ is defined as

$$
K(P)=\{y \in \mathbb{N} \backslash\{0\} \mid \tau(y)<\tau(y+1)\} .
$$

That is, the set of kinks is no longer evaluated in terms of differences between the slope of $P$ at $y$ and at $y+1$, but in terms of differences between the type 
indifferent between consuming $y-1$ or $y$ and that indifferent between $y$ and $y+1$.

Fix $(T, E) \in \mathcal{Q} \mathcal{L}^{1}$ and let $\varphi(T, E)=(\mathbf{x}, \mathbf{y})$ be represented by the standard serial pricing scheme $\left(a^{s}, p^{s}\right)$. Let $\left(\mathbf{x}^{\prime}, \mathbf{y}^{\prime}\right) \in A(T, E)$ be an allocation Paretodominating $(\mathbf{x}, \mathbf{y})$. We prove that $\left(\mathbf{x}^{\prime}, \mathbf{y}^{\prime}\right)$ is not feasible. As in the proof of Theorem 3 , there is no loss of generality in assuming that $p(y) \leq v_{y}^{*}=\arg \max r_{y}\left(v, E_{y}\right)$ for all $y \in \mathbb{N} \backslash\{0\}$.

We need the following immediate corollary to Assumption QL1.

Corollary to Assumption QL1. Let $y, y^{\prime} \in \mathbb{N} \backslash\{0\}$ be such that $y \leq y^{\prime}$, let $\gamma, \gamma^{\prime} \in \mathbb{R}_{++}$be such that $\gamma<\gamma^{\prime} \leq \max _{w \in \mathbb{R}_{+}} r_{y^{\prime}}(w)$, and let $w, w^{\prime}$ be the smallest valuations in $\mathbb{R}_{+}$such that $r_{y}(w)=\gamma$ and $r_{y^{\prime}}\left(w^{\prime}\right)=\gamma^{\prime}$, respectively. Then for each $\Delta \in \mathbb{R}_{+}$such that $0<\Delta \leq w$,

$$
r_{y^{\prime}}\left(w^{\prime}+\Delta\right)-r_{y^{\prime}}\left(w^{\prime}\right)<r_{y}(w)-r_{y}(w-\Delta) .
$$

Let $\bar{y}=\mathbf{y}(\bar{t}), \bar{y}^{\prime}=\mathbf{y}^{\prime}(\bar{t})$ and $\bar{y}^{*}=\min \left\{\bar{y}, \bar{y}^{\prime}\right\}$. We can construct a finite sequence of standard pricing schemes $\left(a^{k}, p^{k}\right), k \in\{0, \ldots, K\}$, such that

(1) $p^{k}(y)=V(y, \bar{t})$ for all $k \in\{0, \ldots, K\}$ and all $y>\bar{y}^{*}$,

(2) $a^{0}=a$ and $p^{0}(y)=p(y)$ for all $y \in\left\{1, \ldots, \bar{y}^{*}\right\}$,

(3) $a^{K}=a^{\prime}$ and $p^{K}(y)=p^{\prime}(y)$ for all $y \in\left\{1, \ldots, \bar{y}^{*}\right\}$,

(4) for all $k \in\{1, \ldots, K\}$, one of the following statements holds:

(a) $a^{k}<a^{k-1}$ and $p^{k}(y)=p^{k-1}(y)$ for all $y \in\left\{1, \ldots, \bar{y}^{*}\right\}$,

(b) $a^{k}<a^{k-1}, p^{k}(1)=p^{k-1}+a^{k-1}-a^{k}$, and $p^{k}(y)=p^{k-1}(y)$ for all $y \in\left\{2, \ldots, \bar{y}^{*}\right\}$,

(c) there exists $y(k) \in\left\{1, \ldots, \bar{y}^{*}\right\}$ such that $p^{k}(y(k))<p^{k-1}(y(k))$ and $p^{k}(y)=p^{k-1}(y)$ for all $y \neq y(k)$,

(d) there exists $y(k) \in\left\{1, \ldots, \bar{y}^{*}\right\}$ and $\Delta \in \mathbb{R}_{+}$such that $p^{k}(y(k))=$ $p^{k-1}(y(k))-\Delta, p^{k}(y(k)+1)=p^{k-1}(y(k)+1)+\Delta$, and $p^{k}(y)=p^{k-1}(y)$ for all $y \neq y(k), y(k)+1$.

For all $k \in\{0, \ldots, K\}$, let $R\left(a^{k}, p^{k}\right)=a^{k}+\sum_{y=1}^{\bar{y}^{*}} r_{y}\left(p^{k}(y)\right)$. We claim that $R\left(a^{k-1}, p^{k-1}\right)>R\left(a^{k}, p^{k}\right)$ for all $k \in\{1, \ldots, K\}$. Indeed, consider the move from $\left(a^{k-1}, p^{k-1}\right)$ to $\left(a^{k}, p^{k}\right)$. If (4)(a) holds, then the claim follows from the equality $R\left(a^{k}, p^{k}\right)=R\left(a^{k-1}, p^{k-1}\right)-\left(a^{k-1}-a^{k}\right)$. If (4)(b) holds, the claim follows from the fact that $r_{1}(p) \leq p$ for all $p \in \mathbb{R}_{+}$. If $(4)(\mathrm{c})$ holds, it follows from the inequalities $p^{k}(y(k)), p^{k-1}(y(k)) \leq v_{y(k)}^{*}$. If $(4)(\mathrm{d})$ holds, it follows from the Corollary to Assumption QL1. 
By definition of serial allocations, $R\left(a^{0}, p^{0}\right)=C(\bar{y})>R\left(a^{K}, p^{K}\right)$. If $\bar{y}^{*}=\bar{y} \leq$ $\bar{y}^{s}$, then $\int_{T} \mathbf{x} d E=R\left(a^{K}, p^{K}\right)$, proving that $(\mathbf{x}, \mathbf{y})$ is not feasible. If $\bar{y}^{*}=\bar{y}^{s}<\bar{y}$, then $\max _{w \in \mathbb{R}_{+}} r_{y}(w)<c(y)$ for all $y>\bar{y}^{*}$ by definition of serial allocations, so that $\int_{T} \mathbf{x} d E<C\left(\bar{y}^{*}\right)+\sum_{y=\bar{y}^{*}+1}^{\mathbf{y}(\bar{t})} p(y)$, showing again that $(\mathbf{x}, \mathbf{y})$ is not feasible.

Part 2. Suppose $\varphi$ satisfies efficiency and demand monotonicity. We show that it is serial. The proof is a simple adaptation of Part 2 of the proof of Theorem 3.

For every $k \in \mathbb{N} \backslash\{0\}$, define

$$
\mathcal{Q} \mathcal{L}^{1}(k)=\left\{(T, E) \in \mathcal{Q} \mathcal{L}^{1} \mid \max _{t \in T} r_{k}\left(v(k, t), E_{k}\right) \leq c(k)\right\} .
$$

The first step of the proof carries over, mutatis mutandis, because the extension to quasi-linear preferences is irrelevant as long as production does not exceed one unit.

The second step is the induction argument. Fix $k \in \mathbb{N} \backslash\{0\}$ and make the induction hypothesis that

for all $(T, E) \in \mathcal{Q L}^{1}(k), \varphi(T, E)$ is a serial allocation for $(T, E)$.

$\operatorname{Fix}(T, E) \in \mathcal{Q L}^{1}(k+1) \backslash \mathcal{Q} \mathcal{L}^{1}(k)$, that is, suppose $c(k)<\max _{t \in T} r_{k}\left(v(k, t), E_{k}\right) \leq$ $c(k+1)$. In order to show that $\varphi(T, E)$ is a serial allocation for $(T, E)$, the key step consists in constructing an economy $\left(T^{1}, E^{1}\right)$ such that $\left(T^{1}, E^{1}\right) \unlhd(T, E)$ and $\max _{t \in T^{1}} r_{k}\left(v(k, t), E_{k}^{1}\right)=c(k)$. This is done as follows.

For $y=1, \ldots, k$, denote by $v^{s}(y)$ the smallest valuation $v \in \mathbb{R}_{+}$such that $r_{y}\left(v, E_{y}\right) \geq c(y)$. Define $\left(T^{1}, E^{1}\right)$ such that $T^{1}=T$ and

$$
\begin{aligned}
E_{k}^{1}(v) & =E_{k}(v) \text { if } v \leq v^{s}(k), \\
& =\min \left\{E_{k}\left(v^{s}(k)\right)+\frac{1}{\varepsilon}\left(v-v^{s}(k)\right),(1-\varepsilon)+\frac{\varepsilon}{v(k, \bar{t})} v, 1\right\} \text { if } v>v^{s}(k) .
\end{aligned}
$$

Remember that, because of the single-crossing property, $T$ is one-dimensional, so that, given $V$, as soon as $E_{k}^{1}($.$) is defined, so is E_{k^{\prime}}^{1}($.$) for all k^{\prime} \neq k$. Consequently, the above requirement uniquely defines $E^{1}$.

Now, the change from $E$ to $E^{1}$ affects the distribution of the valuations of all units of the public good. In order to translate the rest of the proof of Theorem 3 , we must show that the standard serial prices of the first $k$ units remain unaffected.

For $y=1, \ldots, k$, denote by $v^{s}\left(y, E^{1}\right)$ the smallest valuation $v \in \mathbb{R}_{+}$such that $r_{y}\left(v, E_{y}^{1}\right) \geq c(y)$. We need to prove that $v^{s}\left(y, E^{1}\right)=v^{s}(y)$. Let $t^{*} \in T$ be defined by 
$v\left(k, t^{*}\right)=v^{s}(k)$. By construction of $E^{1}, E^{1}(t)=E(t)$ for all $t \leq t^{*}$. Consequently, $r_{y}\left(v(y, t), E^{1}\right)=r_{y}(v(y, t), E)$ for all $t \leq t^{*}$. By definition, for all $y, y^{\prime} \in \mathbb{N} \backslash\{0\}$ such that $y>y^{\prime}, v(y, t) \leq v\left(y^{\prime}, t\right)$ so that $r_{y}(v(y, t), E) \leq r_{y^{\prime}}\left(v\left(y^{\prime}, t\right), E\right)$ for all $t \in T$. But $v^{s}(y) \geq v^{s}\left(y^{\prime}\right)$ for all $y, y^{\prime} \in\{1, \ldots, k\}$ such that $y>y^{\prime}$. Therefore, for each $y \in\{1, \ldots, k\}$, if $t \in T$ is such that $v(y, t)=v^{s}(y)$, then $t \leq t^{*}$, $r_{y}\left(v(y, t), E^{1}\right)=r_{y}(v(y, t), E)$ and $v^{s}\left(y, E^{1}\right)=v(y, t)$, as desired.

The rest of the proof carries over with the obvious notational changes.

\section{References}

[1] Clarke, E. (1971), Multipart pricing of public goods, Public Choice 8, 19-33.

[2] Crawford, V. (1979), A procedure for generating Pareto-efficient egalitarianequivalent allocations, Econometrica 47, 49-60.

[3] Dearden, J. A. (1998), Serial cost sharing of excludable public goods: general cost functions, Economic Theory 12, 189-198.

[4] Deb, R., I. Ghosh and T.K. Seo (2002), The pivotal mechanism for excludable public goods: Characterization and welfare asymptotics, Mathematical Social Sciences 43, 209-224.

[5] Demange, G. (1984), Implementing efficient and egalitarian equivalent allocations, Econometrica 52, 1167-1177.

[6] Diamond, P. (1998), Optimal income taxation: An example with a U-shaped pattern of optimal marginal tax rates, American Economic Review 88, 83-95.

[7] Drèze, J. A. (1980), Public goods with exclusions, Journal of Public Economics 13, 5-24.

[8] Fleurbaey, M. and F. Maniquet (2006), A theory of fairness and social welfare, forthcoming.

[9] Green, J. and J.-J. Laffont (1979), Characterization of satisfactory mechanisms for the revelation of preferences for public goods, Econometrica 45, 427-438.

[10] Groves, T. (1973), Incentives in teams, Econometrica 41, 617-631. 
[11] Groves, T. and J. Ledyard (1977), Optimal allocation of public goods: a solution to the free-rider problem, Econometrica 45, 783-809.

[12] Hellwig, (2005), A utilitarian approach to the provision and pricing of excludable public goods, Journal of Public Economics, 1981-2003.

[13] Hurwicz, L. (1979), Outcome functions yielding Walrasian and Lindahl allocations at Nash equilibrium points, Review of Economic Studies 46, 217-225.

[14] Kaneko, M. (1977), The ratio equilibrium and the core of the voting game $G(N, W)$ in a public goods economy, Econometrica 45, 1589-1594.

[15] Kolm, S.-Ch. (1966), The optimal production of justice, Paper presented at the 1966 conference of the International Economic Association on Public Economics in Biaritz. Published in the proceedings of this conference, Economie Publique CNRS, Paris, 1968, and Public Economics, Macmillan.

[16] Lindahl, E. (1928), Some controversial questions in the theory of taxation, reprinted in Classics in the Theory of Public Finance, (R. A. Musgrave and A. T. Peacock, Eds) St. Martin's Press, New York, 1958.

[17] Maniquet, F. (2003), Implementation of allocation rules under perfect information, Social Choice and Welfare 21, 323-346.

[18] Maniquet, F. and Y. Sprumont (2004), Fair production and allocation of an excludable nonrival good, Econometrica 72, 627-640.

[19] Maniquet, F. and Y. Sprumont (2005), Welfare egalitarianism in nonrival environments, Journal of Economic Theory 120, 155-174.

[20] Mas-Colell, A. (1980), On efficiency and decentralization in the pure theory of public goods, Quarterly Journal of Economics 94, 625-641.

[21] Mirrlees, J. (1971), An exploration in the theory of optimum income taxation, Review of Economic Studies 38, 175-208.

[22] Moulin, H. (1987), Egalitarian-equivalent cost-sharing of a public good, Econometrica 55, 963-977.

[23] Moulin, H. (1994), Serial cost-sharing of excludable public goods, Review of Economic Studies 61, 305-325. 
[24] Moulin, H. and S. Shenker (2001), Strategyproof sharing of submodular costs: budget balance versus efficiency, Economic Theory 18, 511-533.

[25] Mutuswami, S. (2004), Strategyproof cost sharing of a binary good and the egalitarian solution, Mathematical Social Sciences 48, 271-280.

[26] Norman, P. (2004), Efficient mechanisms for public goods with use exclusions, Review of Economic Studies 71, 1163-1188.

[27] Olszewski, W. (2004), Coalition-proof mechanisms for provision of excludable public goods, Games and Economic Behavior 46, 88-114.

[28] Shorrocks, A. F. (1983), Ranking income distributions, Economica 50, 3-17.

[29] Sprumont, Y. (1998), Equal factor equivalence in economies with multiple public goods, Social Choice and Welfare 15, 543-558.

[30] Spence, (1973), Job market signaling, Quarterly Journal of Economics 87, $355-374$.

[31] Thomson, W. (2004), The theory of fair allocation, Princeton University Press, forthcoming.

[32] Walker, M. (1981), A simple incentive compatible scheme for attaining Lindahl allocations, Econometrica 49, 65-71. 
Document 1: Proof that the rule in Example 5 is population-monotonic

Let $(I, E)$ and $\left(I^{\prime}, E^{\prime}\right)$ be two economies such that $(I, E)$ is obtained from $\left(I^{\prime}, E^{\prime}\right)$ by an increase in population. That is, $I \subseteq I^{\prime}$ and $E-E^{\prime}$ is nonnegative and nondecreasing. We prove that $p(1, E) \leq p\left(1, E^{\prime}\right)$ and $p(1, E)+p(2, E) \leq$ $p\left(1, E^{\prime}\right)+p\left(2, E^{\prime}\right)$.

Let $(J, F)$ and $\left(J^{\prime}, F^{\prime}\right)$ be the two sub-economies obtained from $(I, E)$ and $\left(I^{\prime}, E^{\prime}\right)$ according to the construction described in Example 5 . Let $w_{*}, w_{*}^{\prime} \in \mathbb{R}_{+}$be the smallest valuations maximizing $r(., F)$ and $r\left(., F^{\prime}\right)$, respectively. In particular, $r\left(w_{*}, F\right)=r\left(w_{*}^{\prime}, F^{\prime}\right)=C(2)$.

Step 1. $w_{*} \leq w_{*}^{\prime}$.

Assume not. Then

$$
w_{*}^{\prime}\left(m(F)-F\left(w_{*}^{\prime}\right)\right)<C(2)=w_{*}^{\prime}\left(m\left(F^{\prime}\right)-F^{\prime}\left(w_{*}^{\prime}\right)\right),
$$

so that

$$
m(F)-F\left(w_{*}^{\prime}\right)<m\left(F^{\prime}\right)-F^{\prime}\left(w_{*}^{\prime}\right) .
$$

Now, given that $E-E^{\prime}$ is nondecreasing, $F-F^{\prime}$ is also nondecreasing, so that

$$
F\left(w_{*}\right)-F^{\prime}\left(w_{*}\right) \geq F\left(w_{*}^{\prime}\right)-F^{\prime}\left(w_{*}^{\prime}\right)
$$

and therefore,

$$
m(F)-F\left(w_{*}\right)<m\left(F^{\prime}\right)-F^{\prime}\left(w_{*}\right) .
$$

Consequently,

$$
w_{*}\left(m(F)-F\left(w_{*}\right)\right)=C(2)<w_{*}\left(m\left(F^{\prime}\right)-F^{\prime}\left(w_{*}\right)\right),
$$

contradicting the fact that $r\left(., F^{\prime}\right)$ is maximized at $w_{*}^{\prime}$.

As a corollary, $m(F) \geq m\left(F^{\prime}\right)$. This follows from $w_{*} \leq w_{*}^{\prime}, F\left(w_{*}\right) \geq F^{\prime}\left(w_{*}\right)$, and

$$
w_{*}\left(m(F)-F\left(w_{*}\right)\right)=w_{*}^{\prime}\left(m\left(F^{\prime}\right)-F^{\prime}\left(w_{*}^{\prime}\right)\right)>w_{*}\left(m\left(F^{\prime}\right)-F^{\prime}\left(w_{*}\right)\right),
$$

that is, $m(F)-F\left(w_{*}\right)>m\left(F^{\prime}\right)-F^{\prime}\left(w_{*}\right)$.

Step 2. $m(E-F) \geq m\left(E^{\prime}-F^{\prime}\right)$.

Assume not. We know that $r\left(w_{*}^{\prime}, E\right) \geq r\left(w_{*}^{\prime}, E^{\prime}\right)$. Then, $r\left(w_{*}^{\prime}, E^{\prime}\right)=C(2)+$ $w_{*}^{\prime} m\left(E^{\prime}-E\right)$ implies that $r\left(w_{*}^{\prime}, E\right) \geq C(2)+w_{*}^{\prime} m\left(E^{\prime}-F^{\prime}\right)$, so that

$$
C(2) \leq r\left(w_{*}^{\prime}, E\right)-w_{*}^{\prime} m\left(E^{\prime}-E\right)<r\left(w_{*}^{\prime}, E\right)-w_{*}^{\prime} m(E-F) \leq r\left(w_{*}^{\prime}, F\right),
$$


which means that $r(., F)$ is not maximized at $w_{*}$, a contradiction.

Step 3. $p(1, E) \leq p\left(1, E^{\prime}\right)$.

We must show that $\min \left\{v^{s}(1, F), b(E)\right\} \leq \min \left\{v^{s}\left(1, F^{\prime}\right), b\left(E^{\prime}\right)\right\}$.

Since $r(v, E) \geq r\left(v, E^{\prime}\right)$ for all $v \in \mathbb{R}_{+}$, we have $b(E) \leq b\left(E^{\prime}\right)$.

Moreover, $F(v) \geq F^{\prime}(v)$ for all $v \in \mathbb{R}_{+}$, so that $r(v, F) \geq r\left(v, F^{\prime}\right)$ for all $v \in$ $\mathbb{R}_{+}$. This implies that $v^{s}(1, F) \leq v^{s}\left(1, F^{\prime}\right)$ since $r\left(v^{s}(1, F), F\right)=r\left(v^{s}\left(1, F^{\prime}\right), F^{\prime}\right)=$ $C(1)$.

Step 4. $p(1, E)+p(2, E) \leq p\left(1, E^{\prime}\right)+p\left(2, E^{\prime}\right)$.

Let us prove the claim in the case where $p(1, E)=v^{s}(1, F)<p\left(1, E^{\prime}\right)=$ $v^{s}\left(1, F^{\prime}\right) \leq p\left(2, E^{\prime}\right)<p(2, E)$. The case where either $p(1, E)$ or $p\left(1, E^{\prime}\right)$ or both reach their upper bound is dealt with in a similar way. Note first that $p(2, E) \leq w_{*}$. Let us assume that $p(1, E)+p(2, E)=p\left(1, E^{\prime}\right)+p\left(2, E^{\prime}\right)$ and show that

$$
r(p(1, E), E)+r(p(2, E), E) \geq r\left(p\left(1, E^{\prime}\right), E^{\prime}\right)+r\left(p\left(2, E^{\prime}\right), E^{\prime}\right),
$$

so that the claim follows.

We have

$$
\begin{aligned}
r(p(2, E), E) & =r(p(2, E), F)+p(2, E) m(E-F) \text { and } \\
r\left(p\left(2, E^{\prime}\right), E^{\prime}\right) & =r\left(p\left(2, E^{\prime}\right), F\right)+p\left(2, E^{\prime}\right) m\left(E^{\prime}-F^{\prime}\right),
\end{aligned}
$$

whereas

$$
\begin{aligned}
r(p(1, E), E) & =C(1)+p(1, E) m(E-F) \text { and } \\
r\left(p\left(1, E^{\prime}\right), E^{\prime}\right) & =C(1)+p\left(1, E^{\prime}\right) m\left(E^{\prime}-F,\right) .
\end{aligned}
$$

So, (9.27) holds if and only if

$$
\begin{aligned}
& p(1, E) m(E-F)+r(p(2, E), F)+p(2, E) m(E-F) \\
\geq & p\left(1, E^{\prime}\right) m\left(E^{\prime}-F^{\prime}\right)+r\left(p\left(2, E^{\prime}\right), F\right)+p\left(2, E^{\prime}\right) m(E-F),
\end{aligned}
$$

which is true, as $p(1, E)+p(2, E)=p\left(1, E^{\prime}\right)+p\left(2, E^{\prime}\right), r(p(2, E), F) \geq r\left(p\left(2, E^{\prime}\right), F\right)$, and $m(E-F) \geq m\left(E^{\prime}-F^{\prime}\right)$ 


\section{Document 2: Proof of Theorem 5}

Let $(T, E)$ be an economy.

Statement (a). Let $(a, p)$ be a standard pricing scheme for $(T, E)$, with associated nondecreasing type scheme $\tau$, and fix $t \in T$. By (7.1) in the text, $\tau(y) \leq t \leq$ $\tau(y+1)$. Given that $\tau$ is nondecreasing, this implies

$$
\begin{aligned}
& v(y, t) \geq p(y)=v(y, \tau(y)) \text { for all } y \leq \mathbf{y}(t), \\
& v(y, t) \leq p(y)=v(y, \tau(y)) \text { for all } y \geq \mathbf{y}(t) .
\end{aligned}
$$

Combined with (7.2), this yields that, for all $y \in \mathbb{N} \backslash\{0\}$,

$$
\begin{aligned}
V(\mathbf{y}(t), t)-\mathbf{x}(t) & =\sum_{y^{\prime}=1}^{\mathbf{y}(t)}\left(v\left(y^{\prime}, t\right)-p\left(y^{\prime}\right)\right)-a \\
& \geq \sum_{y^{\prime}=1}^{y}\left(v\left(y^{\prime}, t\right)-p\left(y^{\prime}\right)\right)-a \\
& =V(y, t)-\mathbf{x}\left(\mathbf{y}^{-1}(y)\right),
\end{aligned}
$$

where $\mathbf{y}^{-1}(y)$ stands for any $t$ such that $\mathbf{y}(t)=y$.

Statement (b). Let $(\mathbf{x}, \mathbf{y})$ be an incentive-compatible allocation for $(T, E)$. We only show how to construct the standard pricing scheme generating it, and the associated type scheme. The proof that they are unique parallels the proof in the linear case and is omitted.

For any $t, t^{\prime} \in T$, applying (7.1) to both $\left(t, t^{\prime}\right)$ and $\left(t^{\prime}, t\right)$ gives

$$
\sum_{y=\mathbf{y}(t)}^{\mathbf{y}\left(t^{\prime}\right)} v(y, t) \leq \mathbf{x}\left(t^{\prime}\right)-\mathbf{x}(t) \leq \sum_{y=\mathbf{y}(t)}^{\mathbf{y}\left(t^{\prime}\right)} v\left(y, t^{\prime}\right) .
$$

It follows that both $\mathbf{y}$ and $\mathbf{x}$ are non-decreasing functions and $\mathbf{x}(t)=\mathbf{x}\left(t^{\prime}\right)$ if and only if $\mathbf{y}(t)=\mathbf{y}\left(t^{\prime}\right)$. Since $T$ is compact, both $\mathbf{x}$ and $\mathbf{y}$ must have a finite range: there exists $N \in \mathbb{N}$ such that

$$
\begin{aligned}
& \mathbf{y}(T)=\left\{y^{0}, \ldots, y^{N}\right\} \\
& \mathbf{x}(T)=\left\{x^{0}, \ldots, x^{N}\right\}
\end{aligned}
$$

with $y^{n}<y^{n+1}$ and $x^{n}<x^{n+1}$ whenever $n, n+1 \in\{0, \ldots, N\}$, and $\mathbf{x}(t)=x^{n}$ if and only if $\mathbf{y}(t)=y^{n}$. 
For $n=0, \ldots, N$, define $T^{n}=\left\{t \in T \mid(\mathbf{x}(t), \mathbf{y}(t))=\left(x^{n}, y^{n}\right)\right\}$ and $t^{n}=\inf T^{n}$. Note that $\underline{t}=t^{0} \leq \ldots \leq t^{N} \leq \bar{t}$. Moreover, because $E$ is strictly increasing, $t^{n}=\sup T^{n-1}$ for $i=1, \ldots, N$. Applying (9.28) to $t \in T^{n-1}, t^{\prime} \in T^{n}$ and taking limits as $t$ tends to $\sup T^{n-1}$ and $t^{\prime}$ tends to $\inf T^{n}$,

$$
\sum_{y=y^{n-1}+1}^{y^{n}} v\left(y, t^{n}\right)=x^{n}-x^{n-1},
$$

and, therefore, $t^{n-1} \leq t^{n}$.

Define now the standard pricing scheme $(a, p)$ as follows: $p(y)=v\left(y, t^{\min \left\{n \mid y \leq y^{n}\right\}}\right)$ if $1 \leq y \leq y^{N}, p(y)=v(y, \bar{t})$ if $y>y^{N}$ otherwise, and $a=x^{0}-\sum_{y=1}^{y^{0}} v(y, \underline{t})$. By construction, $(a, p)$ satisfies equations (7.1) and (7.2) for every $t \in T$. Let the associated $\tau$ be defined as follows: $\tau(y)=t^{n} \Leftrightarrow p(y)=v\left(y, t^{n}\right)$. 